\title{
A COMBINATORIAL INVARIANT FOR SPHERICAL CR STRUCTURES*
}

\author{
ELISHA FALBEL ${ }^{\dagger}$ AND QINGXUE WANG F $^{\ddagger}$
}

\begin{abstract}
We study a cross-ratio of four generic points of $S^{3}$ which comes from spherical CR geometry. We construct a homomorphism from a certain group generated by generic configurations of four points in $S^{3}$ to the pre-Bloch group $\mathcal{P}(\mathbb{C})$. If $M$ is a 3 -dimensional spherical $\mathrm{CR}$ manifold with a $\mathrm{CR}$ triangulation, by our homomorphism, we get a $\mathcal{P}(\mathbb{C})$-valued invariant for $M$. We show that when applying to it the Bloch-Wigner function, it is zero. Under some conditions on $M$, we show the invariant lies in the Bloch group $\mathcal{B}(k)$, where $k$ is the field generated by the cross-ratio. For a $\mathrm{CR}$ triangulation of the Whitehead link complement, we show its invariant is a torsion in $\mathcal{B}(k)$ and for a triangulation of the complement of the $5_{2}$-knot we show that the invariant is not trivial and not a torsion element.
\end{abstract}

Key words. Bloch group, cross-ratio, spherical CR structure.

AMS subject classifications. 19M05, 57M27, 57M50.

1. Introduction. One can define a number of invariants out of a 3-manifold $M$ equipped with a complete real hyperbolic structure. For instance, by Mostow's rigidity theorem, the volume of a hyperbolic 3-manifold turns out to be a topological invariant of the underlying manifold. Another such invariant is the Chern-Simons invariant $C S(M)$ with values in $\mathbb{R} / \mathbb{Z}([\mathrm{CS}])$. Both invariants can be seen as arising from an invariant associated to a hyperbolic manifold with values in the Bloch group (for the definition see Section 2). The Bloch group $\mathcal{B}(\mathbb{C})$ is a subgroup of the preBloch group $\mathcal{P}(\mathbb{C})$ which is defined as the abelian group generated by all the points in $\mathbb{C} \backslash\{0,1\}$ quotiented by the 5 -term relations. The volume and the Chern-Simons invariant can then be seen through a function (the Bloch regulator)

$$
\mathcal{B}(\mathbb{C}) \rightarrow \mathbb{C} / \mathbb{Q}
$$

The imaginary part being the volume and the real part being $C S(M) \bmod \mathbb{Q}$.

Another geometric structure on 3-manifolds which has been studied for a long time is the Cauchy-Riemann (CR) structure. More precisely, consider $S^{3} \subset \mathbb{C}^{2}$ with the contact structure obtained as the intersection $D=T S^{3} \cap J T S^{3}$ where $J$ is the multiplication by $i$ in $\mathbb{C}^{2}$. The operator $J$ restricted to $D$ defines the standard CR structure on $S^{3}$. The group of CR-automorphisms of $S^{3}$ is $P U(2,1)$ and we say that a manifold $M$ has a spherical $\mathrm{CR}$ structure if it has a $\left(S^{3}, P U(2,1)\right)$ - geometric structure.

A configuration of four points in $S^{3}$ can be thought as defining a CR simplex which can be parametrised through certain cross-ratios. In this paper we obtain an invariant associated to a simplicial complex by $\mathrm{CR}$ simplices with values in the preBloch group $\mathcal{P}(\mathbb{C})$ and with some additional hypothesis, it lies in $\mathcal{B}(\mathbb{C})$. In fact one can define the invariant in $\mathcal{P}(k)$ where $k$ is the field generated by the cross-ratios of the simplicial complex. In the case $k$ is an imaginary quadratic extension of a totally real field and if the invariant is in $\mathcal{B}(k)$, a theorem by Borel will imply that the

\footnotetext{
*Received September 10, 2011; accepted for publication April 10, 2012.

†Institut de Mathématiques, Université Pierre et Marie Curie, 4, Place Jussieu, F-75252, Paris, France (falbel@math.jussieu.fr).

$¥$ School of Mathematical Sciences, Fudan University, Shanghai, 200433, P. R. China (qxwang@ fudan.edu.cn). Q. Wang was supported by NSFC grant \#10801034.
} 
element is torsion and therefore $C S(M)=0$. It is interesting to compare this result with the real hyperbolic geometry case; Neumann and Yang [NY1] proved with the same hypothesis that $C S(M)=0$, although the corresponding element in the Bloch group is never a torsion since the hyperbolic volume is non-vanishing. On the other hand, in Section 5.2, we have examples with invariants which are non-trivial torsions in the Bloch group.

The paper is organized as follows. In the second section, we recall the basic definitions of (pre)-Bloch group, cross-ratio structures, triangulations, and CR geometry. In the third section, we study a complex of configurations of generic points in $S^{3}$, and a homomorphism to the pre-Bloch group $\mathcal{P}(\mathbb{C})$. For a 3 -dimensional spherical $\mathrm{CR}$ manifold $M$ with a given $\mathrm{CR}$ triangulation, we associate a $\mathcal{P}(\mathbb{C})$-valued invariant $[M]$ which is independent of the triangulation. We show that when applying to it the Bloch-Wigner dilogarithm function, it is zero. In the fourth section, we show that when $M$ is closed with holonomy with coefficients in a number field or non-compact with unipotent parabolic boundary holonomy, the invariant $[M]$ lies in the Bloch group $\mathcal{B}(k)$, where $k$ is the field generated by the cross-ratio. For such an $M$, we define its Chern-Simons invariant as the real part of $\rho([M])$, where $\rho$ is the Bloch regulator map. In the last section, we compute the invariant for certain simplicial CR structures on the complement of the figure eight knot and the Whitehead link and the $5_{2}$ knot. The torsion element we find associated to the Whitehead link and figureeight knot (see Section 5) is a very basic torsion element and it is null if we adopt a different definition of the Bloch group (see Remark after Definition 4.3). On the other hand we have a CR triangulation of the complement of the $5_{2}$ whose invariant is an element of infinite order in the Bloch group as shown in the last section.

2. Preliminaries. In this section, we recall the basic definitions and properties of (pre)-Bloch group, cross-ratio structures, triangulations, and CR geometry.

2.1. The Bloch group. We consider an arbitrary field $F$ in the next definition although we will only use $\mathbb{C}, \mathbb{R}$ and number fields in this paper. There are several definitions of the Bloch group in the literature but we will follow conventions of [S1].

Definition 2.1. The pre-Bloch group $\mathcal{P}(F)$ is the quotient of the free abelian group $\mathbb{Z}[F \backslash\{0,1\}]$ by the subgroup generated by the 5-term relations

$$
[x]-[y]+\left[\frac{y}{x}\right]-\left[\frac{1-x^{-1}}{1-y^{-1}}\right]+\left[\frac{1-x}{1-y}\right], \quad \forall x, y \in F \backslash\{0,1\} .
$$

Consider the tensor product $F^{*} \otimes_{\mathbb{Z}} F^{*}$, where $F^{*}$ is the multiplicative group of $F$. It is an abelian group satisfying, for $n$ an integer, $n(a \otimes b)=a^{n} \otimes b=a \otimes b^{n}$.

Let $T=\left\langle x \otimes y+y \otimes x \mid x, y \in F^{*}\right\rangle$ be the subgroup of $F^{*} \otimes_{\mathbb{Z}} F^{*}$ generated by $x \otimes y+y \otimes x$, where $x, y \in F^{*}$.

Definition 2.2. $\bigwedge^{2} F^{*}=\left(F^{*} \otimes_{\mathbb{Z}} F^{*}\right) / T$. For $x, y \in F^{*}$, we will denote by $x \wedge y$ the image of $x \otimes y$ in $\bigwedge^{2} F^{*}$.

Note that, for $x, y \in F^{*}$, we have $x \wedge y=-y \wedge x$ and $2 x \wedge x=0$. But $x \wedge x=0$ is not necessarily true in $\bigwedge^{2} F^{*}$.

DEFINITION 2.3. The Bloch group $\mathcal{B}(F)$ is the kernel of the homomorphism $\delta: \mathcal{P}(F) \rightarrow \bigwedge^{2} F^{*}$, which is defined on generators of $\mathcal{P}(F)$ by $\delta([z])=z \wedge(1-z)$.

When $F=\mathbb{C}, \mathcal{P}(\mathbb{C})([\mathrm{DS}]$ theorem 4.16$)$ and $\mathcal{B}(\mathbb{C})([\mathrm{S} 1])$ are uniquely divisible groups and, in fact, are $\mathbb{Q}$-vector spaces with infinite dimension $([\mathrm{S} 1])$. In particular 
they have no torsion. On the other hand, when the field is $\mathbb{R}$ there exists torsion. In particular, for all $x \in \mathbb{R}-\{0,1\}$, the element $[x]+[1-x] \in \mathcal{B}(\mathbb{R})$ does not depend on $x$ and has order six (see [S1] prop. $1.1 \mathrm{pg} 220$ ).

We will need the following result for $\mathcal{B}(k)$ when $k$ contains a cubic root of unity:

Lemma 2.4. Suppose $k$ is a field containing a cubic root of unity. Then

$$
2[z]=2\left[\frac{1}{1-z}\right], \forall z \in k \backslash\{0,1\} .
$$

Proof. By [S1] Lemma 1.5 (c), we have

$$
2([z]+[1-z])=0 .
$$

From Lemma 1.2 of [S1], we obtain

$$
2\left([1-z]+\left[\frac{1}{1-z}\right]\right)=0 .
$$

Therefore,

$$
2[z]=-2[1-z]=2\left[\frac{1}{1-z}\right] .
$$

Consider the complex conjugation in $\mathbb{C}$ and its extension to an involution:

$$
\sigma: \mathbb{Z}[\mathbb{C} \backslash\{0,1\}] \rightarrow \mathbb{Z}[\mathbb{C} \backslash\{0,1\}] .
$$

As $\sigma$ preserves the 5-term relations, it induces an involution on the pre-Bloch group $\mathcal{P}(\mathbb{C})$ which we will also denote by $\sigma$. Let

$$
\mathcal{P}(\mathbb{C})=\mathcal{P}(\mathbb{C})^{+}+\mathcal{P}(\mathbb{C})^{-}
$$

be the decomposition of $\mathcal{P}(\mathbb{C})$ into the two subgroups preserved by the involution. They are the eigenspaces of $\sigma$ acting on the $\mathbb{Q}$-vector space $\mathcal{P}(\mathbb{C})$. We have $\sigma(z)=z$ for $z \in \mathcal{P}(\mathbb{C})^{+}$and $\sigma(z)=-z$ for $z \in \mathcal{P}(\mathbb{C})^{-}$. Analogously

$$
\mathcal{B}(\mathbb{C})=\mathcal{B}(\mathbb{C})^{+}+\mathcal{B}(\mathbb{C})^{-} .
$$

By [S1, Lemma 1.3], for any $x, y \in F-\{0,1\},[x]+[1-x]=[y]+[1-y]$ in $\mathcal{P}(F)$. That is, the element $[x]+[1-x] \in \mathcal{B}(F)$ is independent of the choice of $x$, hence the following definition makes sense.

Definition 2.5. $c_{F}:=[x]+[1-x] \in \mathcal{B}(F)$, where $x$ is any given element of $F \backslash\{0,1\}$.

Let $K_{*}^{M}(F)$ denote the Milnor K-groups of $F$, and $K_{*}(F)$ denote the Quillen's algebraic K-groups of $F$. It is well-known that $K_{1}^{M}(F) \cong K_{1}(F)=F^{*}$ and $K_{2}^{M}(F) \cong K_{2}(F)$. There is a natural map from $K_{n}^{M}(F)$ to $K_{n}(F)$ for each $n$. The cokernel of this map is called the group of indecomposable elements, denoted by $K_{n}^{\text {ind }}(F)$.

For $n=3$ and $F$ an infinite field, we have the following fundamental exact sequence, due to Suslin ([S1, Theorem 5.2]):

$$
0 \longrightarrow \operatorname{Tor}(\mu(F), \mu(F))^{\sim} \longrightarrow K_{3}^{\text {ind }}(F) \stackrel{s_{F}}{\longrightarrow} \mathcal{B}(F) \longrightarrow 0,
$$


where $\mu(F)$ is the group of roots of unity in $F$. If char $F=2$, $\operatorname{Tor}(\mu(F), \mu(F))^{\sim}=\operatorname{Tor}(\mu(F), \mu(F))$. If $\operatorname{char} F \neq 2$, $\operatorname{Tor}(\mu(F), \mu(F))^{\sim}$ is the unique nontrivial extension of $\operatorname{Tor}(\mu(F), \mu(F))$ by $\mathbb{Z} / 2$, that is, we have the nontrivial extension

$$
0 \longrightarrow \mathbb{Z} / 2 \longrightarrow \operatorname{Tor}(\mu(F), \mu(F))^{\sim} \longrightarrow \operatorname{Tor}(\mu(F), \mu(F)) \longrightarrow 0
$$

For a field extension $E / F$, there are natural homomorphisms $\alpha: K_{3}^{\text {ind }}(F) \rightarrow K_{3}^{\text {ind }}(E)$ and $\beta: \mathcal{B}(F) \rightarrow \mathcal{B}(E)$.

LEMmA 2.6. Let $F$ be an infinite field and $E / F$ a field extension. The homomorphism $s_{F}: K_{3}^{\text {ind }}(F) \rightarrow \mathcal{B}(F)$ in (2) is functorial in $E / F$ with respect to $\alpha, \beta$. That is, we have the following commutative diagram:

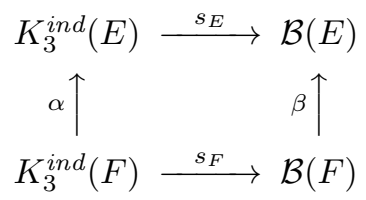

Proof. It follows from the construction of the exact sequence (2) in [S1]. The first step in the construction is the homomorphism from $H_{3}(G L(F))$ to $\mathcal{B}(F)$ via spectral sequences, see [S1, Proposition 3.1, Theorem 4.1]. The second step is the Hurewicz homomophism from $K_{3}(F)$ to $H_{3}(G L(F))$, see [S1, Lemma 5.4, Theorem 5.1]. It is clear that both steps are functorial with respect to the field extension $E / F$. Hence the diagram commutes.

LEMMA 2.7. Let $E$ be a field of characteristic 0. If $\operatorname{Tor}(\mu(E), \mu(E))$ has no element of order 3 , then $2 c_{E} \in B(E)$ has order 3 .

Proof. Denote by $o(g)$ the order of a group element $g$. Consider the field extension $E / \mathbb{Q}$. By $\left[\mathrm{S} 1\right.$, Corollary 5.3], the Bloch group $\mathcal{B}(\mathbb{Q})$ is generated by $c_{\mathbb{Q}}$ and $o\left(c_{\mathbb{Q}}\right)=6$. Hence $o\left(2 c_{\mathbb{Q}}\right)=3$. By Lemma 2.6, we have the commutative diagram:

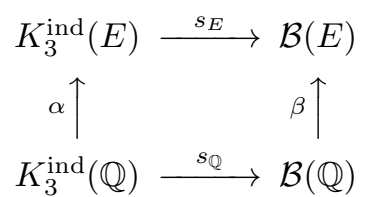

By definition $\beta\left(2 c_{\mathbb{Q}}\right)=2 c_{E}$. Since $o\left(2 c_{\mathbb{Q}}\right)=3, o\left(2 c_{E}\right)=1$ or 3 . It suffices to show that $2 c_{E} \neq 0$. Since $\mu(\mathbb{Q})=\mathbb{Z} / 2, \operatorname{Tor}(\mathbb{Z} / 2, \mathbb{Z} / 2)=\mathbb{Z} / 2$. Hence $\operatorname{Tor}(\mu(\mathbb{Q}), \mu(\mathbb{Q}))^{\sim}=\mathbb{Z} / 4$. Now by the fundamental exact sequence $(2)$, there is a nonzero element $q \in K_{3}^{\text {ind }}(\mathbb{Q})$ such that $s_{\mathbb{Q}}(q)=2 c_{\mathbb{Q}}$. Since $o\left(2 c_{\mathbb{Q}}\right)=3$, we see that $3 \mid o(q)$. Let $r=\alpha(q)$. By the above commutative diagram, we get $s_{E}(r)=2 c_{E}$. Therefore, it suffices to show that $r \notin$ ker $s_{E}$. Indeed, since $\operatorname{Tor}(\mu(E), \mu(E))$ has no element of order 3 , we see that $\operatorname{Tor}(\mu(E), \mu(E))^{\sim}$ does not have an element of order 3 either. Hence it has no element of order divided by 3. By [MS, Proposition 11.3], the homomorphism $\alpha$ is injective. Therefore, $3 \mid o(r)=o(q)$. Now the fundamental exact sequence (2) implies that $r \notin \operatorname{ker} s_{E}$. Hence $o\left(2 c_{E}\right)=3$.

Consider now a number field $k$, that is, an extension of $\mathbb{Q}$ of degree $d<\infty$. We have that $k=\mathbb{Q}(t)$, where $t \in \mathbb{C}$ satisfies an irreducible polynomial with coefficients in $\mathbb{Q}$ with degree $d$. Each root of the irreducible polynomial determines a field embedding 
$\sigma: k \rightarrow \mathbb{C}$. There are $r_{1}$ real embeddings (when $\sigma(k) \subset \mathbb{R}$ ) and $r_{2}$ pairs of complex conjugate embeddings with $d=r_{1}+2 r_{2}$.

DeFInition 2.8. The Bloch-Wigner function is

$$
D(x)=\arg (1-x) \log |x|-\operatorname{Im}\left(\int_{0}^{x} \log (1-t) \frac{d t}{t}\right) .
$$

It is well-defined and real analytic on $\mathbb{C}-\{0,1\}$ and extends to a continuous function on $\mathbb{C} P^{1}$ by defining $D(0)=D(1)=D(\infty)=0$. It is well-known that it satisfies the 5 -term relation. Hence it gives rise to a well-defined map:

$$
D: \mathcal{P}(k) \rightarrow \mathbb{R},
$$

given by

$$
D\left(\sum_{i=1}^{k} n_{i}\left[x_{i}\right]\right)=\sum_{i=1}^{k} n_{i} D\left(x_{i}\right) .
$$

Generalizing Dirichlet's units theorem, Borel [Bo] proved the following description of $\mathcal{B}(k)$. His results are more general and describe the higher K-theory of number fields. The relation to the Bloch group and the use of the dilogarithm is due to work of Dupont, Sah, Bloch and Suslin. In the following we let $\sigma_{i}, \bar{\sigma}_{i}, 1 \leq i \leq r_{2}$, be the $r_{2}$ pairs of complex embeddings.

Theorem $2.9([\mathrm{Bo}])$. Consider the map $r: \mathcal{B}(k) \rightarrow \mathbb{R}^{r_{2}}$ given by

$$
[z] \rightarrow\left(D\left(\sigma_{1}(z)\right), \cdots, D\left(\sigma_{r_{2}}(z)\right)\right) .
$$

Then $\operatorname{Im}(r)$ is a lattice in $\mathbb{R}^{r_{2}}$ and $\operatorname{Ker}(r)$ is the torsion subgroup of $\mathcal{B}(k)$.

Recall that we have the Bloch regulator map:

$$
\rho: \mathcal{B}(\mathbb{C}) \rightarrow \mathbb{C} / \pi^{2} \mathbb{Q}=\mathbb{R} / \pi^{2} \mathbb{Q} \oplus i \mathbb{R}
$$

It is known that the imaginary part of $\rho$ coincides with the Bloch-Wigner function $D$.

2.2. Tetrahedra with cross-ratio structures. In the following we recall the definition of cross-ratio structures of [F3].

Consider a set of four elements $\Delta=\left\{p_{0}, p_{1}, p_{2}, p_{3}\right\}$. We call $p_{i}, 0 \leq i \leq 3$ the vertices of $\Delta$. Let $O \Delta$ be the set of all orderings of $\Delta$. We will denote an element of $O \Delta$ by $\left[p_{i}, p_{j}, p_{k}, p_{l}\right]$ (where $\{i, j, k, l\}=\{0,1,2,3\}$ ) and call it a simplex although we only deal with configurations of four points. Given $\Delta$, there are 24 simplices divided in two classes $O \Delta^{+}$(containing $\left.\left[p_{0}, p_{1}, p_{2}, p_{3}\right]\right)$ and $O \Delta^{-}$(containing $\left.\left[p_{0}, p_{1}, p_{3}, p_{2}\right]\right)$ of 12 elements each. Each class is an orbit of the even permutation group acting on $O \Delta$.

The following definition assigns similarity invariants to each vertex of a configuration of four points.

Definition 2.10. A cross-ratio structure on a set of four points $\Delta=\left\{p_{0}, p_{1}, p_{2}, p_{3}\right\}$ is a function defined on the ordered quadruples

$$
\mathbf{X}: O \Delta \rightarrow \mathbb{C} \backslash\{0,1\}
$$

satisfying, if $(i, j, k, l)$ is any permutation of $(0,1,2,3)$, the relations 
1.

$$
\mathbf{X}\left(p_{i}, p_{j}, p_{k}, p_{l}\right)=\frac{1}{\mathbf{X}\left(p_{i}, p_{j}, p_{l}, p_{k}\right)} .
$$

2. (similarity relations)

$$
\mathbf{X}\left(p_{i}, p_{j}, p_{k}, p_{l}\right)=\frac{1}{1-\mathbf{X}\left(p_{i}, p_{l}, p_{j}, p_{k}\right)} .
$$

REMARKS.

1. To visualize the definition we refer to Figure 1 . For each $\left[p_{i}, p_{j}, p_{k}, p_{l}\right] \in O \Delta^{+}$ we define

$$
z_{i j}=\mathbf{X}\left(p_{i}, p_{j}, p_{k}, p_{l}\right)
$$

We interpret $z_{i j}$ as a cross-ratio associated to the edge $[i j]$ at the vertex $i$. Cross-ratios of elements of $O \Delta^{-}$are obtained taking inverses by the first symmetry. In the following we shall denote by a sequence of numbers $(i j k l)$ the corresponding invariant $\mathbf{X}\left(u_{i}, u_{j}, u_{k}, u_{l}\right)$.

2. The similarity relations can be used to reduce the number of variables to four, one for each vertex. One can use, for instance, $\left(z_{01}, z_{10}, z_{23}, z_{32}\right) \in(\mathbb{C} \backslash\{0,1\})^{4}$.

3. If we impose that

$$
z_{01}=z_{10}=z_{23}=z_{32},
$$

we may interpret the configuration of four points as a configuration of points in $\mathbb{C} P^{1}$ with $z_{01}$ one of the cross-ratios.

2.3. Triangulations, cross-ratio structures and an invariant. A triangulation of a manifold is an explicit description of it as a simplicial complex. Equivalence of triangulations was studied in $[\mathrm{A}]$. Alexander defined certain moves on triangulations and showed that any two triangulations of a closed manifold are related by a sequence of these moves. A particularly simple description of that equivalence was obtained by Pachner $[\mathrm{P}]$ through some elementary moves. In particular, Pachner proved that any two triangulations are obtained from each other through the following two moves and their inverses.

Definition 2.11. An elementary (or Pachner) move in a complex defined by a triangulation is

- (2-3 move) the substitution of two simplices $\left[u_{0}, u_{1}, u_{2}, u_{3}\right]-\left[u_{0}, u_{1}, u_{2}, u_{4}\right]$, which have a common face, by three simplices $-\left[u_{0}, u_{1}, u_{3}, u_{4}\right]+\left[u_{0}, u_{2}, u_{3}, u_{4}\right]-\left[u_{1}, u_{2}, u_{3}, u_{4}\right]$ with one common edge and vice-versa.

- (1-4 move) the substitution of a simplex $\left[u_{0}, u_{1}, u_{2}, u_{3}\right]$ by four simplices

$$
\left[u_{0}, u_{1}, u_{2}, u_{4}\right]-\left[u_{0}, u_{1}, u_{3}, u_{4}\right]+\left[u_{0}, u_{2}, u_{3}, u_{4}\right]-\left[u_{1}, u_{2}, u_{3}, u_{4}\right]
$$

by adding a new vertex $u_{4}$ and vice-versa.

To deal with manifolds with boundary it is useful to introduce ideal triangulations. It is important in the following to consider singular triangulations, meaning that we allow self or multiple intersections between simplices (along 2-faces). 


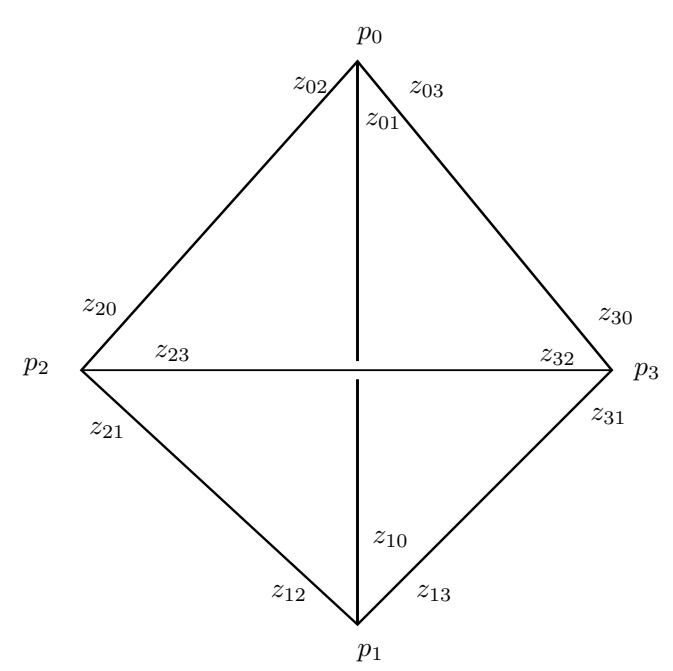

FIG. 1. Parameters for a cross-ratio structure

Definition 2.12. Let $M$ be a compact manifold with boundary $\partial M$. Denote by $\hat{M}$ the manifold obtained by collapsing each boundary component. An ideal triangulation is a singular triangulation of $\hat{M}$ such that its vertices are the points of $\hat{M}$ obtained from collapsing the boundary components of $M$.

A related concept we will not deal with in this paper is that of a spine of a 3 -manifold with boundary. Ideal triangulations are in natural bijection to standard spines and existence and equivalence of ideal triangulations were proven through the corresponding existence and equivalence theorems for standard spines (a general reference is $[\mathrm{Ma} 1])$. We will need the following folklore theorem (cf. [Ma, P]).

THEOREM 2.13. Any two ideal triangulations of a manifold with boundary can be obtained from one another through the 2-3 moves.

DEFINITION 2.14. Let $T$ be a (ideal, if the 3-manifold is not closed) triangulation of a 3-manifold. Let $\mathbf{X}\left(p_{i}, p_{j}, p_{k}, p_{l}\right)$ be a cross-ratio structure defined on the simplices. The pair $(T, \mathbf{X})$ is called a cross-ratio structure associated to a triangulation if the following compatibility conditions are satisfied:

1. Edge compatibility: If $\left[p_{i}, p_{j}, p_{m_{0}}, p_{m_{1}}\right],\left[p_{i}, p_{j}, p_{m_{1}}, p_{m_{2}}\right], \cdots,\left[p_{i}, p_{j}, p_{m_{n}}, p_{m_{0}}\right]$ are simplices having the edge $\left[p_{i}, p_{j}\right]$ in common then

$$
\mathbf{X}\left(p_{i}, p_{j}, p_{m_{0}}, p_{m_{1}}\right) \cdots \mathbf{X}\left(p_{i}, p_{j}, p_{m_{n}}, p_{m_{0}}\right)=1
$$

2. Face compatibility: If $\left[p_{i}, p_{j}, p_{k}, p_{l}\right]$ and $\left[p_{i^{\prime}}, p_{j}, p_{k}, p_{l}\right]$ are two simplices with 
a common face $\left[p_{j}, p_{k}, p_{l}\right]$ then

$$
\begin{aligned}
& \mathbf{X}\left(p_{j}, p_{i}, p_{k}, p_{l}\right) \mathbf{X}\left(p_{k}, p_{i}, p_{l}, p_{j}\right) \mathbf{X}\left(p_{l}, p_{i}, p_{j}, p_{k}\right) \\
= & \mathbf{X}\left(p_{j}, p_{i^{\prime}}, p_{k}, p_{l}\right) \mathbf{X}\left(p_{k}, p_{i^{\prime}}, p_{l}, p_{j}\right) \mathbf{X}\left(p_{l}, p_{i^{\prime}}, p_{j}, p_{k}\right) .
\end{aligned}
$$

DeFINITION 2.15. To each cross-ratio structure associated to a triangulation as above we define the element

$$
\beta(T, \mathbf{X})=\sum_{s}\left(\left[z_{01}^{s}\right]+\left[z_{10}^{s}\right]+\left[z_{12}^{s}\right]+\left[z_{21}^{s}\right]\right) \in \mathcal{P}(\mathbb{C})
$$

where $s$ indexes the simplices in the triangulation and $z_{i j}^{s}$ are the cross-ratios of the simplex $s$.

Consider $\left(T^{\prime}, \mathbf{X}^{\prime}\right)$ and $(T, \mathbf{X})$, two cross-ratio structures on two triangulations obtained from each other by an elementary move. Given the five vertices $\{i, j, k, l, m\}=\{0,1,2,3,4\}$, where the move is concentrated as above, we write $(i j k l)$ the cross-ratio defined by these points (which might be $\mathbf{X}^{\prime}(i j k l)$ or $\mathbf{X}(i j k l)$ according to which triangulation the simplex $[i j k l]$ belongs).

Definition 2.16. We say that $\left(T^{\prime}, \mathbf{X}^{\prime}\right)$ is obtained from $(T, \mathbf{X})$ by an elementary move if the triangulation $T^{\prime}$ is obtained from $T$ by an elementary move and the following relations are satisfyed:

1. edge compatibility conditions

$$
(i j k l)=(i j k m)(i j m l) .
$$

2. face compatibility conditions

$$
(i j k l)(l j i k)(k j l i)=(i m k l)(l m i k)(k m l i) .
$$

TheOREM 2.17. If $\left(T^{\prime}, \mathbf{X}^{\prime}\right)$ is obtained from $(T, \mathbf{X})$ by an elementary move then $\beta\left(T^{\prime}, \mathbf{X}^{\prime}\right)=\beta(T, \mathbf{X})$.

Proof. It is exactly the proof of Theorem 5.2 in [F3].

2.4. CR geometry (see [C, BS, G, J]). CR geometry is modeled on the Heisenberg group $\mathfrak{N}$, the set of pairs $(z, t) \in \mathbb{C} \times \mathbb{R}$ with the product

$$
(z, t) \cdot\left(z^{\prime}, t^{\prime}\right)=\left(z+z^{\prime}, t+t^{\prime}+2 \operatorname{Im} z \bar{z}^{\prime}\right) .
$$

The one point compactification of the Heisenberg group, $\overline{\mathfrak{N}}$, of $\mathfrak{N}$ can be interpreted as $S^{3}$ which, in turn, can be identified to the boundary of complex hyperbolic space.

We consider the group $U(2,1)$ preserving the Hermitian form $\langle z, w\rangle=w^{*} J z$ defined on $\mathbb{C}^{3}$ by the matrix

$$
J=\left(\begin{array}{lll}
0 & 0 & 1 \\
0 & 1 & 0 \\
1 & 0 & 0
\end{array}\right)
$$

and the following subspaces in $\mathbb{C}^{2,1}$ (that is, $\mathbb{C}^{3}$ with the above Hermitian form).

$$
V_{0}=\left\{z \in \mathbb{C}^{3}-\{0\} \quad: \quad\langle z, z\rangle=0\right\},
$$




$$
V_{-}=\left\{z \in \mathbb{C}^{3}:\langle z, z\rangle<0\right\} .
$$

Let $\mathbb{P}: \mathbb{C}^{3} \backslash\{0\} \rightarrow \mathbb{C} P^{2}$ be the canonical projection. Then $\mathbf{H}_{\mathbb{C}}^{2}=\mathbb{P}\left(V_{-}\right)$is the complex hyperbolic space and $S^{3}=\partial \mathbf{H}_{\mathbb{C}}^{2}=\mathbb{P}\left(V_{0}\right)$ can be identified to $\overline{\mathfrak{N}}$.

The group of biholomorphic transformations of $\mathbf{H}_{\mathbb{C}}^{2}$ is then $P U(2,1)$, the projectivization of $U(2,1)$. It acts on $S^{3}$ by $\mathrm{CR}$ transformations. We define $\mathbb{C}$-circles as boundaries of complex lines in $\mathbf{H}_{\mathbb{C}}^{2}$. Analogously, $\mathbb{R}$-circles are boundaries of totally real totally geodesic two dimensional submanifolds in $\mathbf{H}_{\mathbb{C}}^{2}$. Using the identification $S^{3}=\mathfrak{N} \cup\{\infty\}$, one can define alternatively a $\mathbb{C}$-circle as any circle in $S^{3}$ which is obtained from the vertical line $\{(0, t) \in \mathfrak{N} \mid t \in \mathbb{R}\} \cup\{\infty\}$ in the compactified Heisenberg space by translation by an element of $P U(2,1)$. Analogously, $\mathbb{R}$-circles are all obtained by translations of the horizontal line $\{(x, 0) \in \mathfrak{N} \mid x \in \mathbb{R}\} \cup\{\infty\}$.

A point $p=(z, t)$ in the Heisenberg group and the point $\infty$ are lifted to the following points in $\mathbb{C}^{2,1}$ :

$$
\hat{p}=\left[\begin{array}{c}
\frac{-|z|^{2}+i t}{2} \\
z \\
1
\end{array}\right] \quad \text { and } \quad \hat{\infty}=\left[\begin{array}{l}
1 \\
0 \\
0
\end{array}\right] .
$$

Consider $\mathbb{C}^{2,1}$ with the Hermitian form defined by $J$. The Hermitian structure defines a Hermitian form on $\Lambda^{3}\left(\mathbb{C}^{2,1}\right)$. In fact, let $\left\{e_{1}, e_{2}, e_{3}\right\}$ be a basis of $\mathbb{C}^{2,1}$ such that

$$
\left\langle e_{1}, e_{1}\right\rangle=\left\langle e_{2}, e_{2}\right\rangle=-\left\langle e_{3}, e_{3}\right\rangle=1 .
$$

Then $e=e_{1} \wedge e_{2} \wedge e_{3}$ is a basis of $\Lambda^{3}\left(\mathbb{C}^{2,1}\right)$ and we define $\left\langle e_{1} \wedge e_{2} \wedge e_{3}, e_{1} \wedge e_{2} \wedge e_{3}\right\rangle=-1$.

Definition 2.18. Let $v, w \in \mathbb{C}^{2,1}$. We define the Hermitian cross-product $v \otimes w$ by the formula

$$
\langle u, v \otimes w\rangle e=u \wedge v \wedge w,
$$

for all $u \in \mathbb{C}^{2,1}$.

In coordinates, $v=\left(v_{0}, v_{1}, v_{2}\right)$ and $w=\left(w_{0}, w_{1}, w_{2}\right)$, we compute

$$
v \otimes w=\left(\bar{v}_{0} \bar{w}_{1}-\bar{v}_{1} \bar{w}_{0}, \bar{v}_{2} \bar{w}_{0}-\bar{v}_{0} \bar{w}_{2}, \bar{v}_{1} \bar{w}_{2}-\bar{v}_{2} \bar{w}_{1}\right) .
$$

So we observe that the coordinates of $v \otimes w$ are in the field generated by the conjugates of the coordinates of $v$ and $w$.

Definition 2.19. Given any three ordered points $p_{0}, p_{1}, p_{2} \in \partial \mathbf{H}_{\mathbb{C}}^{2}$, we define Cartan's angular invariant $\mathbb{A}$ as

$$
\mathbb{A}\left(p_{0}, p_{1}, p_{2}\right)=\arg \left(-\left\langle\hat{p}_{0}, \hat{p}_{1}\right\rangle\left\langle\hat{p}_{1}, \hat{p}_{2}\right\rangle\left\langle\hat{p}_{2}, \hat{p}_{0}\right\rangle\right) .
$$

Triple of points in $S^{3}$ are classified by their Cartan invariant according to the following proposition by Cartan (see $[\mathrm{G}]$ ).

Proposition 2.20. Two ordered triples of pairwise distinct points $\left(p_{0}, p_{1}, p_{2}\right)$ and $\left(p_{0}^{\prime}, p_{1}^{\prime}, p_{2}^{\prime}\right)$ in $\partial \mathbf{H}_{\mathbb{C}}^{2}$ are equivalent under an element of $P U(2,1)$ if and only if

$$
\mathbb{A}\left(p_{0}, p_{1}, p_{2}\right)=\mathbb{A}\left(p_{0}^{\prime}, p_{1}^{\prime}, p_{2}^{\prime}\right) .
$$


Definition 2.21. We say that $n(n \geq 1)$ points in $S^{3}$ are generic if they are distinct and, if $n \geq 3$, any three of them are not contained in a $\mathbb{C}$-circle.

As the action of $P U(2,1)$ is doubly transitive, a simple computation gives the following normalization for a triple of generic points.

LEMMA 2.22. One can always normalize a triple $\left(p_{0}, p_{1}, p_{2}\right)$ of generic points in $S^{3}$ so that in the compactified Heisenberg group they are

$$
p_{0}=\infty \quad p_{1}=(0,0) \quad p_{2}=(1, t) \quad \text { with } t \in \mathbb{R} .
$$

In that case $\tan \mathbb{A}\left(p_{0}, p_{1}, p_{2}\right)=t$.

2.5. Configurations of four points. We refer to Figure 1 to describe the parameters of a tetrahedron (see also [F2, F3]). Consider a generic configuration of four (ordered) points $\left(p_{0}, p_{1}, p_{2}, p_{3}\right)$ in $S^{3}$. Fix one of them say $p_{0}$ and consider the projective space of complex lines passing through it. Then $p_{1}, p_{2}, p_{3}$ determine three points $t_{1}, t_{2}, t_{3}$ on $\mathbb{C} P^{1}$. The fourth point corresponds to the complex line passing through $p_{0}$ and tangent to $S^{3}$, call it $t_{0}$. The cross-ratio of those four points in $\mathbb{C} P^{1}$ is $z_{01}=\mathbf{X}\left(t_{0}, t_{1}, t_{2}, t_{3}\right)$ (here, $\mathbf{X}$ is the usual cross-ratio of four points in $\left.\mathbb{C} P^{1}\right)$. We define analogously the other invariants $z_{i j}, 0 \leq i, j \leq 3$. If we take $p_{0}=\infty$, the complex lines passing through $p_{0}$ intersect $\mathfrak{N}$ in vertical lines which are then determined by a coordinate in $\mathbb{C}$. Up to Heisenberg translations, we can assume that $p_{1}=(0,0)$ and $p_{2}=\left(1, s_{2}\right)$ and $p_{3}=\left(z_{01}, s_{3}\right), s_{2}, s_{3} \in \mathbb{R}$. The corresponding points in $\mathbb{C} P^{1}$ will be $\infty, 0,1, z_{01}$. Therefore one "sees" at the vertex $p_{0}$ the Euclidean triangle determined by $0,1, z_{01} \in \mathbb{C}$.

We associate to each vertex $i$, in the edge $[i j]$, the invariant $(i j k l)$ where the order of $k$ and $l$ is fixed by the right hand rule with the thumb pointed from $j$ to $i$. A shortcut notation for the invariants is therefore

$$
z_{i j}=(i j k l)
$$

the indices $k l$ being determined by the choice $i j$.

In order to give an explicit formula (see [W2, Ge]), denote by $\hat{p}$ a lift of $p \in S^{3}$.

Definition 2.23. Given two points $p_{i}$ and $p_{j}$ in $S^{3}$, a polar vector $c_{i j}$ is a perpendicular vector to the complex line defined by $p_{i}$ and $p_{j}$.

Observe that a polar vector is given by solving the equations $\left\langle\hat{p}_{i}, c_{i j}\right\rangle=\left\langle\hat{p}_{j}, c_{i j}\right\rangle=0$ and therefore one can choose a polar vector to have coordinates in the field defined by the conjugates of the coordinates of $\hat{p}_{i}$ and $\hat{p}_{j}$.

DEFINITION 2.24. Consider a generic configuration of four (ordered) points $\left(p_{0}, p_{1}, p_{2}, p_{3}\right)$ in $S^{3}$. For $\left[p_{i}, p_{j}, p_{k}, p_{l}\right] \in \Delta O^{+}$, a positively oriented configuration, define

$$
z_{i j}=(i j l k)=\frac{\left\langle\hat{p}_{l}, c_{i j}\right\rangle\left\langle\hat{p}_{k}, \hat{p}_{i}\right\rangle}{\left\langle\hat{p}_{k}, c_{i j}\right\rangle\left\langle\hat{p}_{l}, \hat{p}_{i}\right\rangle} .
$$

It satisfies the relations $(i j l k)=(i j k l)^{-1}$ and

$$
z_{i j} z_{j i}=\overline{z_{k l} z_{l k}} .
$$


Moreover, we have the following description of the space of configurations of four points.

Proposition 2.25. (cf. [F2]) Configurations (up to translations by $P U(2,1)$ ) of four generic points in $S^{3}$ are parametrised by

$$
V \subset\left(\mathbb{C}^{*} \backslash\{1\}\right)^{12}
$$

with coordinates $z_{i j}, 1 \leq i \neq j \leq 4$, defined by, for $(i, j, k, l)$ an even permutation of $(0,1,2,3)$, the usual similarity constraints

$$
z_{i k}=\frac{1}{1-z_{i j}}
$$

and the three complex equations

$$
z_{i j} z_{j i}=\overline{z_{k l} z_{l k}}
$$

with the exclusion of solutions such that $z_{i j} z_{j i} z_{i k} z_{k i} z_{i l} z_{l i}=-1$ and $z_{i j} z_{j i} \in \mathbb{R}$.

REMARK. The real solutions parametrise configurations with four points contained in an $\mathbb{R}$-circle. The solutions such that $z_{i j} z_{j i} z_{i k} z_{k i} z_{i l} z_{l i}=-1$ and $z_{i j} z_{j i} \in \mathbb{R}$ correspond to degenerate hyperbolic ideal tetrahedra with four points contained in the boundary of a totally geodesic plane in real hyperbolic space. They don't correspond to CR tetrahedra. See [Ge] for more details and [F1, W1, W2, PP, PP1, FP] for other descriptions.

We can also describe generic configurations of four points in $S^{3}$ by the following Lemma (see [F2, Proposition 4.3]).

LEMMA 2.26. One can always normalize a quadruple $\left(p_{0}, p_{1}, p_{2}, p_{3}\right)$ of generic points in $S^{3}$ so that in the compactified Heisenberg group they are

$$
p_{0}=\infty \quad p_{1}=(0,0) \quad p_{2}=(1, t) \quad p_{3}=\left(z, s|z|^{2}\right),
$$

where $(z, t, s)$ are in the set

$$
K=\left\{(z, s, t) \in \mathbb{C} \times \mathbb{R} \times \mathbb{R} \mid z \neq 0,1 \text { and } \bar{z} \frac{s+i}{t+i} \neq 1\right\} .
$$

In that case, the invariants of the configuration are

$$
z_{01}=z, z_{10}=\frac{\bar{z}(s+i)}{t+i}, z_{23}=\frac{z[(t+i)-\bar{z}(s+i)]}{(z-1)(t-i)}, z_{32}=\frac{\bar{z}(z-1)(s-i)}{(t+i)-\bar{z}(s+i)} .
$$

So the set $V$ in the above proposition is homeomorphic to the set $K$ defined above.

DeFinition 2.27. To each configuration of four generic points $\left(p_{0}, p_{1}, p_{2}, p_{3}\right)$, we define an element

$$
\beta\left(p_{0}, p_{1}, p_{2}, p_{3}\right):=\left[z_{01}\right]+\left[z_{10}\right]+\left[z_{23}\right]+\left[z_{32}\right]
$$

in $\mathcal{P}(\mathbb{C})$, the pre-Bloch group. 
3. A $\mathcal{P}(\mathbb{C})$-valued CR invariant. In this section, let $G=P U(2,1)$. For $n \geq 0$, we define $C_{n}\left(S^{3}\right)$ to be the free abelian group generated by the set of all generic $(n+1)$ ordered points in $S^{3}$ (see Definition 2.21).

The group $G$ acts on $S^{3}$ and therefore it acts diagonally on $C_{n}\left(S^{3}\right)$, which gives $C_{n}\left(S^{3}\right)$ a left $G$-module structure.

We define the differential $d_{n}: C_{n}\left(S^{3}\right) \rightarrow C_{n-1}\left(S^{3}\right)$ by

$$
d_{n}\left(p_{0}, \ldots, p_{n}\right)=\sum_{i=0}^{n}(-1)^{i}\left(p_{0}, \ldots, \hat{p}_{i}, \ldots, p_{n}\right),
$$

then we can check that every $d_{n}$ is a $G$-module homomorphism and $d_{n} \circ d_{n+1}=0$. Hence we have the $G$-complex

$$
C_{\bullet}\left(S^{3}\right): \cdots \rightarrow C_{n}\left(S^{3}\right) \rightarrow C_{n-1}\left(S^{3}\right) \rightarrow \cdots \rightarrow C_{0}\left(S^{3}\right) .
$$

We define the augmentation map $\epsilon: C_{0}\left(S^{3}\right) \rightarrow \mathbb{Z}$ by $\epsilon(x)=1$ for each $x \in S^{3}$.

LEMmA 3.1 (cf. [S1] pg. 221). The augmentation complex $C_{\bullet}\left(S^{3}\right) \rightarrow \mathbb{Z} \rightarrow 0$ is exact.

Proof. Let $C_{-1}\left(S^{3}\right)=\mathbb{Z}$. For $z=\sum_{i=1}^{m} n_{i}\left(p_{0}^{i}, \ldots, p_{k}^{i}\right) \in \operatorname{ker} d_{k}$, we can choose a point $p \in S^{3}$ such that if $k>0, p, p_{0}^{i}, \ldots, p_{k}^{i}$ are generic for all $i$; if $k=0, p, p_{0}^{i}$ are distinct for all $i$. Hence $\left(p, p_{0}^{i}, \ldots, p_{k}^{i}\right) \in C_{k+1}\left(S^{3}\right)$ and we can check directly that $d\left(\sum_{i=1}^{m} n_{i}\left(p, p_{0}^{i}, \ldots, p_{k}^{i}\right)\right)=z$.

For a left $G$-module $M$, we denote $M_{G}$ its group of co-invariants, that is,

$$
M_{G}=M /\langle g m-m, \forall g \in G, m \in M\rangle,
$$

where $\langle g m-m, \forall g \in G, m \in M\rangle$ is the submodule of $M$ generated by all the elements of the form $g m-m, g \in G, m \in M$. Take the co-invariants of the complex $C_{\bullet}\left(S^{3}\right)$, we get the induced complex:

$$
C_{\bullet}\left(S^{3}\right)_{G}: \cdots \rightarrow C_{n}\left(S^{3}\right)_{G} \rightarrow C_{n-1}\left(S^{3}\right)_{G} \rightarrow \cdots \rightarrow C_{0}\left(S^{3}\right)_{G}
$$

with differential $\bar{d}_{n}: C_{n}\left(S^{3}\right)_{G} \rightarrow C_{n-1}\left(S^{3}\right)_{G}$ induced by $d_{n}$. Since $G$ acts double transitively on $S^{3}$, we see that $C_{n}\left(S^{3}\right)_{G}=\mathbb{Z}$ if $n \leq 1$, and the differential $\bar{d}_{1}: C_{1}\left(S^{3}\right)_{G} \rightarrow C_{0}\left(S^{3}\right)_{G}$ is zero. The equivalence class of three generic points under the action of $G$ is determined by their Cartan invariant (see Proposition 2.20), so we get

$$
C_{2}\left(S^{3}\right)_{G}=\mathbb{Z}[\mathbb{R}]
$$

Explicitly, a triple $\left(p_{0}, p_{1}, p_{2}\right) \in C_{2}\left(S^{3}\right)$ determines an element $t=\tan \mathbb{A}\left(p_{0}, p_{1}, p_{2}\right) \in \mathbb{Z}[\mathbb{R}]$. In normalized coordinates, $(\infty, 0,(1, t)) \in C_{2}\left(S^{3}\right)$ is represented by $t \in \mathbb{Z}[\mathbb{R}]$. The differential

$$
\bar{d}_{2}: C_{2}\left(S^{3}\right)_{G} \rightarrow C_{1}\left(S^{3}\right)_{G}=\mathbb{Z}
$$

is given on generators by $\bar{d}_{2}\left(p_{0}, p_{1}, p_{2}\right)=1$.

By Lemma 2.26, we can describe $C_{3}\left(S^{3}\right)_{G}$ as follows:

$$
C_{3}\left(S^{3}\right)_{G}=\mathbb{Z}[K]
$$


where

$$
K=\left\{(z, s, t) \in \mathbb{C} \times \mathbb{R} \times \mathbb{R} \mid z \neq 0,1 ; \bar{z} \frac{s+i}{t+i} \neq 1\right\} .
$$

In normalized coordinates, $\left(\infty, 0,(1, t),\left(z, s|z|^{2}\right)\right) \in C_{3}\left(S^{3}\right)$ is represented by $(z, s, t) \in \mathbb{Z}[K]$, so that

$$
C_{3}\left(S^{3}\right)_{G}=\bigoplus_{(z, s, t) \in K} \mathbb{Z} \cdot\left(\infty, 0,(1, t),\left(z, s|z|^{2}\right)\right) .
$$

By $\mathbb{Z}$-linear extension of Definition 2.27 to $C_{3}\left(S^{3}\right)_{G}$, we have a homomorphism

$$
\bar{\beta}: C_{3}\left(S^{3}\right)_{G} \rightarrow \mathcal{P}(\mathbb{C})
$$

given by

$$
\sum_{l=1}^{k} n_{l}\left(p_{0}^{l}, p_{1}^{l}, p_{2}^{l}, p_{3}^{l}\right) \mapsto \sum_{l=1}^{k} n_{l} \beta\left(\left(p_{0}^{l}, p_{1}^{l}, p_{2}^{l}, p_{3}^{l}\right)\right)
$$

Lemma 3.2. $\bar{\beta}\left(\operatorname{Im}\left(\bar{d}_{4}\right)\right)=0$ in $\mathcal{P}(\mathbb{C})$.

Proof. We need to show that the images are in the subgroup generated by the 5 -term relations. This is a special case of Theorem 2.17 and follows from Theorem 5.2 in [F3].

Therefore, $\bar{\beta}$ induces a well-defined homomorphism:

$$
c: \frac{C_{3}\left(S^{3}\right)_{G}}{\operatorname{Im}\left(\bar{d}_{4}\right)} \rightarrow \mathcal{P}(\mathbb{C})
$$

defined by

$$
c\left(\left[p_{0}, p_{1}, p_{2}, p_{3}\right]\right)=\bar{\beta}\left(p_{0}, p_{1}, p_{2}, p_{3}\right),
$$

where $\left[p_{0}, p_{1}, p_{2}, p_{3}\right]$ denotes the equivalence class of $\left(p_{0}, p_{1}, p_{2}, p_{3}\right)$ in the quotient group. When we restrict to $H_{3}\left(C_{\bullet}\left(S^{3}\right)_{G}\right)$, we get a homomorphism:

$$
c: H_{3}\left(C \bullet\left(S^{3}\right)_{G}\right) \rightarrow \mathcal{P}(\mathbb{C}) .
$$

REMARK 3.3. In the real hyperbolic case, we have $G=P S L_{2}(\mathbb{C})$ acting on $\mathbb{C} P^{1}$ and the corresponding $G$-complex. In that case, we know that $H_{3}\left(C \cdot\left(\mathbb{C} P^{1}\right)_{P S L_{2}(\mathbb{C})}\right)$ is equal to $\mathcal{P}(\mathbb{C})$.

Normalizing the four points $p_{0}=\infty, p_{1}=0, p_{2}=(1, t), p_{3}=\left(z, s|z|^{2}\right)$ according to Lemma 2.26, we obtain the following invariants;

$$
z_{01}=z, z_{10}=\frac{\bar{z}(s+i)}{t+i}, z_{23}=\frac{z[(t+i)-\bar{z}(s+i)]}{(z-1)(t-i)}, z_{32}=\frac{\bar{z}(z-1)(s-i)}{(t+i)-\bar{z}(s+i)}
$$

and the homomorphism $c: \frac{C_{3}\left(S^{3}\right)_{G}}{\operatorname{Im}\left(d_{4}\right)} \rightarrow \mathcal{P}(\mathbb{C})$ can be expressed as

$$
c\left(\left[p_{0}, p_{1}, p_{2}, p_{3}\right]\right)=\left[z_{01}\right]+\left[z_{10}\right]+\left[z_{23}\right]+\left[z_{32}\right] .
$$


Definition 3.4. Given a configuration $\left[p_{0}, p_{1}, p_{2}, p_{3}\right] \in C_{3}\left(S^{3}\right)_{G}$, we say it is symmetric if it lies in the kernel of $\bar{d}_{3}: C_{3}\left(S^{3}\right)_{G} \rightarrow C_{2}\left(S^{3}\right)_{G}$.

Note that this definition is related to the definition of a symmetric tetrahedron introduced in [F2, Section 4.3]. There, a configuration $\left[p_{0}, p_{1}, p_{2}, p_{3}\right]$ is symmetric if there exists an anti-holomorphic involution $\varphi$ such that $\varphi\left(p_{i}\right)=p_{j}$ and $\varphi\left(p_{k}\right)=p_{l}$ for $\{i, j, k, l\}=\{0,1,2,3\}$. Three possible symmetries may appear, namely $\varphi\left(p_{0}\right)=p_{1}$, $\varphi\left(p_{0}\right)=p_{3}$ or $\varphi\left(p_{0}\right)=p_{3}$. They are characterized by $\mathbb{A}\left(p_{0}, p_{1}, p_{2}\right)=\mathbb{A}\left(p_{0}, p_{1}, p_{3}\right)$, $\mathbb{A}\left(p_{0}, p_{2}, p_{3}\right)=\mathbb{A}\left(p_{0}, p_{1}, p_{3}\right)$ and $\mathbb{A}\left(p_{0}, p_{1}, p_{2}\right)=-\mathbb{A}\left(p_{0}, p_{2}, p_{3}\right)$ respectively. But the definition above concerns only the first two of them as shown in the next Lemma.

LEMmA 3.5. $\left[p_{0}, p_{1}, p_{2}, p_{3}\right] \in C_{3}\left(S^{3}\right)_{G}$ is symmetric if and only if the Cartan invariants satisfy

$$
\mathbb{A}\left(p_{0}, p_{1}, p_{2}\right)=\mathbb{A}\left(p_{0}, p_{1}, p_{3}\right) \text { or } \mathbb{A}\left(p_{0}, p_{2}, p_{3}\right)=\mathbb{A}\left(p_{0}, p_{1}, p_{3}\right) .
$$

Proof. By the definition,

$$
\bar{d}_{3}\left(\left[p_{0}, p_{1}, p_{2}, p_{3}\right]\right)=\left[p_{1}, p_{2}, p_{3}\right]-\left[p_{0}, p_{2}, p_{3}\right]+\left[p_{0}, p_{1}, p_{3}\right]-\left[p_{0}, p_{1}, p_{2}\right] .
$$

Hence it lies in kernel of $\bar{d}_{3}$ if and only if in $C_{2}\left(S^{3}\right)_{G}$, we have either

$$
\left[p_{1}, p_{2}, p_{3}\right]=\left[p_{0}, p_{2}, p_{3}\right], \text { and }\left[p_{0}, p_{1}, p_{3}\right]=\left[p_{0}, p_{1}, p_{2}\right]
$$

or

$$
\left[p_{1}, p_{2}, p_{3}\right]=\left[p_{0}, p_{1}, p_{2}\right], \text { and }\left[p_{0}, p_{2}, p_{3}\right]=\left[p_{0}, p_{1}, p_{3}\right]
$$

We know that in $C_{2}\left(S^{3}\right)_{G}$, two elements are equal if and only if their Cartan invariants are the same. It is known that the Cartan invariants satisfy the following cocycle conditions ([G, Page 219]:

$$
\mathbb{A}\left(p_{1}, p_{2}, p_{3}\right)-\mathbb{A}\left(p_{0}, p_{2}, p_{3}\right)+\mathbb{A}\left(p_{0}, p_{1}, p_{3}\right)-\mathbb{A}\left(p_{0}, p_{1}, p_{2}\right)=0 .
$$

Now the lemma follows.

Proposition 3.6. Given $\left[p_{0}, p_{1}, p_{2}, p_{3}\right] \in C_{3}\left(S^{3}\right)_{G}$ as above. Then it is symmetric if and only if one of the following two equivalent conditions holds:

(1). $t=s$ or $t+s-2(s \cdot \operatorname{Re}(z)+\operatorname{Im}(z))=0$;

(2). $\left|z_{01}\right|=\left|z_{32}\right|$.

Proof. By [F2, Proposition 4.6], we have

$$
\tan \mathbb{A}\left(p_{1}, p_{2}, p_{3}\right)=\frac{2(s-t) \operatorname{Re} z+2(1+t s) \operatorname{Im} z+t\left(1+s^{2}\right)|z|^{2}-s\left(1+t^{2}\right)}{|(s-i) z+t-i|^{2}}
$$

and

$$
\tan \mathbb{A}\left(p_{0}, p_{2}, p_{3}\right)=\frac{|z|^{2} s-t+2 \operatorname{Im} z}{|z-1|^{2}}, \tan \mathbb{A}\left(p_{0}, p_{1}, p_{3}\right)=s, \tan \mathbb{A}\left(p_{0}, p_{1}, p_{2}\right)=t .
$$

By Lemma 3.5, we see that it is symmetric if and only if $t=s$ or $s=\frac{|z|^{2} s-t+2 \operatorname{Im} z}{|z-1|^{2}}$, which is condition (1). By the definition of $z_{01}$ and $z_{32}$, a direct computation shows that $\left|z_{01}\right|^{2}=\left|z_{32}\right|^{2}$ if and only if $(t-s)(t+s-2(s \cdot \operatorname{Re}(z)+\operatorname{Im}(z)))=0$. 
Recall that

$$
\mathcal{P}(\mathbb{C})=\mathcal{P}(\mathbb{C})^{+}+\mathcal{P}(\mathbb{C})^{-}
$$

is the decomposition of $\mathcal{P}(\mathbb{C})$ into the two subgroups preserved by the complex conjugation involution.

Proposition 3.7. (1). The image of the homomorphism $c: H_{3}\left(C_{\bullet}\left(S^{3}\right)_{G}\right) \rightarrow \mathcal{P}(\mathbb{C})$ contains $\mathcal{P}(\mathbb{C})^{+}$, the invariant subgroup of $\mathcal{P}(\mathbb{C})$ under complex conjugation.

(2). Suppose $\left[p_{0}, p_{1}, p_{2}, p_{3}\right] \in C_{3}\left(S^{3}\right)_{G}$ is symmetric. Then its image under the homomorphism $c$ lies in $\mathcal{P}(\mathbb{C})^{+}$.

Proof. For (1), we show that the subgroup generated by the images of the symmetric elements contains $\mathcal{P}(\mathbb{C})^{+}$. Consider the elements $\left[p_{0}, p_{1}, p_{2}, p_{3}\right]$ of the form

$$
p_{0}=\infty, p_{1}=0, p_{2}=(1, t), p_{3}=\left(z, t|z|^{2}\right) ; z \in \mathbb{C}-\{0,1\}, t \in \mathbb{R} \text {. }
$$

By Proposition 3.6, they are symmetric. By [F2, Corollary 4.11], we find the invariants:

$$
z_{01}=z, z_{10}=\bar{z}, z_{23}=z e^{i \theta}, z_{32}=\bar{z} e^{-i \theta} ; \quad z \in \mathbb{C}-\{0,1\}, \theta \in \mathbb{R}
$$

Therefore, we have

$$
c\left(\left[p_{0}, p_{1}, p_{2}, p_{3}\right]\right)=[z]+[\bar{z}]+\left[z e^{i \theta}\right]+\left[\bar{z} e^{-i \theta}\right]
$$

By $\left[\right.$ Sah, Theorem 4.16], $\mathcal{P}(\mathbb{C})$ is a $\mathbb{Q}$-vector space. Let $B=\sum_{i=1}^{k} n_{i}\left[a_{i}\right] \in \mathcal{P}(\mathbb{C})^{+}$. Then $\sigma(B)=\sum_{i=1}^{k} n_{i}\left[\overline{a_{i}}\right]=B$. Hence

$$
B=\frac{1}{2}(B+\sigma(B))=\frac{1}{2} \sum_{i=1}^{k} n_{i}\left(\left[a_{i}\right]+\left[\overline{a_{i}}\right]\right)
$$

Choose $b_{i} \in \mathbb{C}$ such that $a_{i}=b_{i}^{2}$ and therefore $\overline{a_{i}}={\overline{b_{i}}}^{2}$. By [DS, Theorem 5.23], we know that in $\mathcal{P}(\mathbb{C}),\left[a^{2}\right]=2([a]+[-a])$. Therefore,

$$
B=\sum_{i=1}^{k} n_{i}\left(\left[b_{i}\right]+\left[\overline{b_{i}}\right]+\left[-b_{i}\right]+\left[-\overline{b_{i}}\right]\right) .
$$

Now in (4), if we choose $z=b_{i}$ and $e^{i \theta}=-1$, we see that the first part follows.

For $(2)$, let $T=c\left(\left[p_{0}, p_{1}, p_{2}, p_{3}\right]\right)=\left[z_{01}\right]+\left[z_{10}\right]+\left[z_{23}\right]+\left[z_{32}\right]$. Using the 5 -term relations, a direct computation shows that in $\mathcal{P}(\mathbb{C})$, the difference $\sigma(T)-T$ is equal to

$$
\left[\frac{z_{32}\left(1-z_{10}\right)}{\bar{z}_{01}\left(1-\bar{z}_{23}\right)}\right]+\left[\frac{\bar{z}_{32}\left(1-\bar{z}_{23}\right)}{z_{01}\left(1-z_{10}\right)}\right]+\left[\frac{z_{01}\left(1-z_{23}\right)}{\bar{z}_{32}\left(1-\bar{z}_{10}\right)}\right]+\left[\frac{\bar{z}_{01}\left(1-\bar{z}_{10}\right)}{z_{32}\left(1-z_{23}\right)}\right]
$$

Put

$$
a=\frac{z_{32}\left(1-z_{10}\right)}{\bar{z}_{01}\left(1-\bar{z}_{23}\right)}, b=\frac{\bar{z}_{32}\left(1-\bar{z}_{23}\right)}{z_{01}\left(1-z_{10}\right)}
$$


we can rewrite

$$
\sigma(T)-T=[a]+[b]+\left[(\bar{a})^{-1}\right]+\left[(\bar{b})^{-1}\right], \text { and } a b=\frac{\left|z_{32}\right|^{2}}{\left|z_{01}\right|^{2}} .
$$

Since $\left[p_{0}, p_{1}, p_{2}, p_{3}\right]$ is symmetric, by Proposition $3.6, a b=1$, i.e. $b=a^{-1}$. On the other hand, we know in $\mathcal{P}(\mathbb{C})$,

$$
[z]+\left[z^{-1}\right]=0
$$

therefore

$$
\sigma(T)-T=[a]+\left[a^{-1}\right]+\left[(\bar{a})^{-1}\right]+[\bar{a}]=0 .
$$

That is, $\sigma(T)=T$ and the image lies in $\mathcal{P}(\mathbb{C})^{+}$. $\mathbf{\square}$

REMARK 3.8. The previous proposition shows that the homomorphism $c: H_{3}\left(C_{\bullet}\left(S^{3}\right)_{G}\right) \rightarrow \mathcal{P}(\mathbb{C})$ is non-trivial, and $\mathcal{P}(\mathbb{C})^{+}$is equal to the image of the subgroup generated by the symmetric configurations. It would be very interesting to determine its kernel and image.

Let $M$ be a 3-dimensional spherical $\mathrm{CR}$ manifold (possibly non-compact). Suppose it has a triangulation consisting of finitely many CR tetrahedra, say $M=\Delta_{1} \cup \cdots \cup \Delta_{k}$ with each $\Delta_{i}$ a CR tetrahedron (here we suppose that the triangulation is ideal if the manifold is non-compact). Denote $p_{0}^{i}, p_{1}^{i}, p_{2}^{i}, p_{3}^{i}$ the four vertices of $\Delta_{i}$ where the order of the vertices is consistent with the orientation.

Definition 3.9. Let $M$ be as above with the $C R$ triangulation. Define an element $[M] \in \frac{C_{3}\left(S^{3}\right)_{G}}{\operatorname{Im}\left(d_{4}\right)}$ by

$$
[M]:=\sum_{i=1}^{k}\left[p_{0}^{i}, p_{1}^{i}, p_{2}^{i}, p_{3}^{i}\right] .
$$

LEMMA 3.10. 1. $[M]$ is independent of the triangulation.

2. $[M] \in H_{3}\left(C \bullet\left(S^{3}\right)_{G}\right)$, that is, $\bar{d}_{3}([M])=0$.

Proof. 1. If $M$ is closed, we know that two different triangulations can be obtained from one to the other by Pachner moves. If $M$ is not closed, by Theorem 2.13, the same result holds. From the definition, it is clear one Pachner move gives an element of $\operatorname{Im}\left(\bar{d}_{4}\right)$. Hence $[M]$ is independent of the triangulation.

2. Since $M$ is triangulated, their faces are matched and the terms in $\bar{d}_{3}([M])$ are canceled out in pairs.

In the following definition we suppose that a triangulation of a non-compact manifold is ideal.

Definition 3.11. Let $M$ be a 3-dimensional spherical $C R$ manifold with a $C R$ triangulation. We define $\beta(M):=c([M]) \in \mathcal{P}(\mathbb{C})$.

Now recall the Bloch-Wigner function (See Definition 2.8) $D: \mathcal{P}(\mathbb{C}) \rightarrow \mathbb{R}$.

Theorem 3.12. Let $M$ be as in the definition 3.11. Then $D(\beta(M))=0$. 
Proof. For a configuration $\left[p_{0}, p_{1}, p_{2}, p_{3}\right] \in C_{3}\left(S^{3}\right)_{G}$ with its cross-ratios $z_{i j}$, by remark 3 of section 6 in [F3, page 14], we have the following identity:

$$
-e^{2 i \mathbb{A}\left(p_{i}, p_{j}, p_{k}\right)}=z_{i l} z_{j l} z_{k l},
$$

where $[i, j, k, l]$ is an even permutation of $[0,1,2,3]$. By [F3, Proposition 6.5], we obtain:

$$
\begin{aligned}
2 D\left(c\left(\left[p_{0}, p_{1}, p_{2}, p_{3}\right]\right)\right)= & D\left(-e^{2 i \mathbb{A}\left(p_{1}, p_{2}, p_{3}\right)}\right)+D\left(-e^{2 i \mathbb{A}\left(p_{0}, p_{3}, p_{2}\right)}\right) \\
& +D\left(-e^{2 i \mathbb{A}\left(p_{0}, p_{1}, p_{3}\right)}\right)+D\left(-e^{2 i \mathbb{A}\left(p_{0}, p_{2}, p_{1}\right)}\right) .
\end{aligned}
$$

Let $M=\Delta_{1} \cup \cdots \cup \Delta_{k}$ with each $\Delta_{i}$ a CR tetrahedron. Denote $p_{0}^{i}, p_{1}^{i}, p_{2}^{i}, p_{3}^{i}$ the four vertices of $\Delta_{i}$ and the order of the vertices is consistent with the orientation. Then by definition

$$
D(\beta(M))=\sum_{i=1}^{k} D\left(c\left(\left[p_{0}^{i}, p_{1}^{i}, p_{2}^{i}, p_{3}^{i}\right]\right)\right) .
$$

Since $M$ is glued by the tetrahedra $\Delta_{i}$, their faces are glued in pairs, and the corresponding Cartan invariants are equal. By the above formula of $2 D\left(c\left(\left[p_{0}, p_{1}, p_{2}, p_{3}\right]\right)\right)$, we see that the terms in $D(\beta(M))$ are canceled in pairs. Hence it is zero.

Remark 3.13. Note that $D(\beta(M))$ is the CR volume of $M$ defined in [F3]. The above theorem says that it is always zero. This is exactly the opposite to the real hyperbolic case where the volume is never zero.

4. An invariant in $\mathcal{B}(k)$. In this section, we will discuss when the $\mathcal{P}(\mathbb{C})$-valued invariant defined above can be defined in the Bloch group $\mathcal{B}(k)$ for $k$ a number field. We first discuss the real hyperbolic case, where it is known that the invariant always lies in $\mathcal{B}(\mathbb{C})$.

Observe first that given a cross-ratio structure $(T, \mathbf{X})$ associated to a triangulation we may associate a field $k_{\mathrm{x}}=\mathbb{Q}\left(z_{1}, \cdots\right)$, where $z_{1}, \cdots$ are all the cross-ratios. It is clearly preserved by taking finite coverings of the structure. From Proposition 4.2 in [F3] we obtain:

\section{Proposition 4.1. The field $k_{\mathbf{x}}$ is invariant under elementary moves.}

For the case of an ideal real hyperbolic triangulation see [NR]. In particular, one can compare $k_{\mathrm{x}}$ to a holonomy representation defined by an ideal triangulation. Recall that the invariant trace field of a representation $\rho: \Gamma \rightarrow P S L(2, \mathbb{C})$ is given by taking lifts $\tilde{g} \in S L(2, \mathbb{C})$ of elements $g \in P S L(2, \mathbb{C})$ and defining $([\mathrm{R}])$

$$
k_{\rho}=\mathbb{Q}\left(\left\{\operatorname{Tr}\left(\tilde{g}^{2}\right) \mid g \in \Gamma\right\}\right) .
$$

For an ideal triangulation of a finite volume cusped hyperbolic manifold, the field obtained by adjoining the cross-ratios of the ideal tetrahedra and the field obtained from a holonomy representation $\rho$ are the same, that is $k_{\rho}=k_{\mathbf{x}}$ ([NR] Theorem $2.4 \mathrm{pg}$. 277). Moreover, if we choose one tetrahedron with one of its faces normalized to be, in homogeneous coordinates of $\mathbb{C} P^{1},[1,0],[0,1],[1,1]$ (that is, $\infty, 0,1$ in $\mathbb{C} \cup\{\infty\}$ ), then the coordinates $\left[z_{i}, 1\right]$ of the vertices of the other tetrahedra obtained by developing the triangulation are all in the field $k_{\mathrm{x}}$ and any side pairing $g \in P S L(2, \mathbb{C})$ which identifies two sides of the triangulation has a lift with coefficients in the same field (see [NR] lemma 2.5 pg. 278). 
In the case of CR structures analogous results were proven in [Ge1]. Denote by $\tilde{g} \in S U(2,1)$ a lift of an element $g \in P U(2,1)$. Let the invariant trace field of a representation $\rho: \Gamma \rightarrow P U(2,1)$ be defined as (see [Ge1, Mc])

$$
k_{\rho}=\mathbb{Q}\left(\left\{\operatorname{Tr}\left((\tilde{\rho}(\gamma))^{3}\right) \mid \gamma \in \Gamma\right\}\right) .
$$

For an ideal triangulation of a CR structure, we can develop it in $S^{3}$ by choosing one tetrahedron with one of its faces normalized to be, in homogeneous coordinates of $\mathbb{C} P^{2}$,

$$
[1,0,0],[0,0,1],[(-1+i t) / 2,1+i t, 1]
$$

(that is, $\infty, 0,1+i t$ in the compactified Heisenberg space $\overline{\mathfrak{N}}$ ), then the coordinates $\left[z_{i}^{1}, z_{i}^{2}, 1\right]$ of the vertices of the other tetrahedra obtained by developing the triangulation are all in the field $k_{\mathrm{x}}$. We will call such a construction a normalized development.

Proposition 4.2 ([Ge]). If $(T, \mathbf{X})$ is a $C R$ triangulation then

$$
k_{\mathbf{x}}=\mathbb{Q}\left(z_{i}^{1}, z_{i}^{2}, \bar{z}_{i}^{1}, \bar{z}_{i}^{2}\right)
$$

where $\left[z_{i}^{1}, z_{i}^{2}, 1\right]$ are as above, the coordinates of the vertices of a normalized development. In particular, $k_{\mathbf{x}}$ is invariant under complex conjugation.

Choosing an edge in each simplex allows us to obtain an element in the pre-Bloch group. We will denote by $T_{b}$ the triangulation with a choice of edge in each simplex. This can, for instance, be achieved by a choice of branching of the triangulation.

Definition 4.3. Let $\left(T_{b}, \mathbf{X}\right)$ be a cross-ratio structure on a triangulation with a choice of edge on each simplex. Define

$$
\beta_{k}\left(T_{b}, \mathbf{X}\right)=\sum_{s}\left(\left[z_{01}^{s}\right]+\left[z_{10}^{s}\right]+\left[z_{23}^{s}\right]+\left[z_{32}^{s}\right]\right) \in \mathcal{P}\left(k_{\mathbf{x}}\right)
$$

REMARK. This definition depends on a choice of edge in each simplex of a triangulation. There are several ways to make it independent of the choice. We can use Lemma 2.4 and define it to be $2 \beta(T, \mathbf{X})$ in $\mathcal{P}\left(k_{\mathbf{x}}(\omega)\right)$, where $\omega$ is a cubic root of unity. We will use, instead, the following

Definition 4.4. Let $(T, \mathbf{X})$ be a cross-ratio structure. Define

$$
\begin{aligned}
& \tilde{\beta}_{k}(T, \mathbf{X})=\sum_{s} \sum_{i \neq j}\left[z_{i j}^{s}\right] \\
& =\sum_{s}\left(\left[z_{01}^{s}\right]+\left[z_{10}^{s}\right]+\left[z_{23}^{s}\right]+\left[z_{32}^{s}\right]+\left[z_{02}^{s}\right]+\left[z_{20}^{s}\right]+\left[z_{13}^{s}\right]+\left[z_{31}^{s}\right]+\left[z_{03}^{s}\right]+\left[z_{30}^{s}\right]+\left[z_{12}^{s}\right]+\left[z_{21}^{s}\right]\right) \in \mathcal{P}\left(k_{\mathbf{X}}\right) .
\end{aligned}
$$

Another definition that is independent of the particular choice of edges in each tetrahedron starts by defining a slightly smaller pre-Bloch group $\mathcal{P}^{\prime}\left(k_{\mathbf{X}}\right)$ as in $([\mathrm{NY}]$ Definition 2.3 pg. 4) to be the quotient of the free abelian group $\mathbb{Z}\left[k_{\mathbf{x}} \cup \infty\right]$ by the subgroup generated by 5 -term relations and

$$
[1]=[0]=[\infty]=0
$$

Both definitions of the pre-Bloch group will differ by a torsion subgroup. With the definition above we have $[z]=\left[\frac{1}{1-z}\right]$ and therefore one can define $\sum_{s}\left(\left[z_{01}^{s}\right]+\left[z_{10}^{s}\right]+\left[z_{23}^{s}\right]+\left[z_{32}^{s}\right]\right)$ as an invariant which does not depend on the choice of a pair of edges in each tetrahedron. 
4.1. The real hyperbolic invariant. We will show for a finite volume noncompact real hyperbolic 3-manifold with ideal triangulation, the corresponding invariant lies in $B(\mathbb{C})$. This follows from $[\mathrm{NZ}]$ (see a proof in $[\mathrm{NY}]$ ). But we give an elementary geometric proof of the identity which will give an idea of the proof in the CR case.

Consider an ideal triangulation of a 3-manifold. We order the simplices by choosing arbitrarily a first one and then a second one with a common face. Having chosen $n$ simplices we choose the $(n+1)$-th as a simplex with one face in common with the union of the previous ones or, if there are two common faces, they share a common edge (if there are three common faces, the edges defined by each pair should be common too). In this way we obtain a polyhedron homeomorphic to a 3-ball with face pairings on its boundary (homeomorphic to a sphere).

Suppose the triangulation has a hyperbolic cross-ratio structure. To each order on the simplices as above, we can associate a well defined map from the 0-skeleton of each simplex to $\mathbb{C} P^{1}$. We can associate with the first simplex the points $\infty, 0,1, z$ and proceed determining the other vertices which are clearly defined by the cross-ratios. In fact, the map is determined by the chosen order on the simplices and the initial map defined on the 0 -skeleton of the first simplex.

Definition 4.5. The holonomy group of the hyperbolic structure is the group generated by the face pairing transformations of the polyhedron.

Let $p_{i} \in \mathbb{C} P^{1}$ be the vertices of the development map as above. Choose a lift $v_{i} \in \mathbb{C}^{2}$ for each vertex $p_{i}$. The vertices of the faces of the polyhedron are identified by side pairings in $\operatorname{PSL}(2, \mathbb{C})$ but their lifts to $\mathbb{C}^{2}$ might not be identified by lifts of the side pairings to $S L(2, \mathbb{C})$.

DEFINITION 4.6. We call a lift of the vertices of a development of a triangulation special if there are lifts of the side pairings which preserve the lifted vertices up to multiplication by -1 .

LEMMA 4.7. Any ideal triangulation of a finite volume 3-manifold has a special lift.

Proof. We consider a finite ideal triangulation of the 3-manifold and its development by ideal tetrahedra in hyperbolic space. We are only interested in its vertices in $\mathbb{C} P^{1}$. Let $\Gamma \in P S L(2, \mathbb{C})$ be the holonomy group of the hyperbolic structure.

Choose one vertex $p \in \mathbb{C} P^{1}$ and a lift $v \in \mathbb{C}^{2}$ of $p$. Without loss of generality, we suppose that $v=(1,0)(p=\infty)$. The elements of $S L(2, \mathbb{C})$ which are lifts of elements of the parabolic group fixing $p$ are of the form

$$
\pm\left[\begin{array}{cc}
1 & \star \\
0 & 1
\end{array}\right]
$$

Consider all vertices identified to $p$ by the holonomy group. That is, the other vertices $p_{i}$ are obtained by $p_{i}=g_{i} p$ for $g_{i} \in \Gamma$. Let $\hat{g}_{i} \in S L(2, \mathbb{C})$ be a lift of $g_{i}$ and let $v_{i}=\hat{g}_{i} v$. We first observe that the definition is compatible up to a multiplication by -1 . Indeed, if $g_{k} p=g_{l} p$ then $g_{l}^{-1} g_{k} p=p$ and therefore $g_{l}^{-1} g_{k}$ is parabolic (or the identity) and $\hat{g}_{k} v= \pm \hat{g}_{l} v$.

Let $g \in \Gamma$ be a side pairing of the polyhedra obtained by the development map. We have $g g_{i} p=g_{j} p$, therefore $\left(g_{j}\right)^{-1} g g_{i} p=p$ and we conclude that $\left(g_{j}\right)^{-1} g g_{i}$ is 
parabolic and then its lift $\left(\hat{g}_{j}\right)^{-1} \hat{g} \hat{g}_{i}$ is a parabolic element (or the identity element) fixing $v$ up to sign. This implies that $\hat{g} \hat{g}_{i} v= \pm \hat{g}_{j} v$ and we conclude that

$$
\hat{g} v_{i}= \pm v_{j} .
$$

We do that for each cycle of vertices and obtain that the lift is special.

Here we use the following observation in [DZ]. Let $v_{i}=\left(v_{i}^{1}, v_{i}^{2}\right) \in \mathbb{C}^{2}$ for $0 \leq i \leq 3$ and define the determinant

$$
\left[v_{i}, v_{j}\right]=\left|\begin{array}{cc}
v_{i}^{1} & v_{j}^{1} \\
v_{i}^{2} & v_{j}^{2}
\end{array}\right| .
$$

Suppose $\left(v_{0}, v_{1}, v_{2}, v_{3}\right)$ is a quadruple of points in $\mathbb{C}^{2}$ so that they define a quadruple of pairwise distinct points $\left(p_{0}, p_{1}, p_{2}, p_{3}\right)$ in $\mathbb{C} P^{1}$, where $h\left(v_{i}\right)=p_{i}$ is the projection in projective space. Observe that

$$
\left[p_{0}, p_{1}, p_{2}, p_{3}\right]=\frac{\left[v_{2}, v_{0}\right]\left[v_{3}, v_{1}\right]}{\left[v_{3}, v_{0}\right]\left[v_{2}, v_{1}\right]}
$$

is the cross ratio of the projection of the four distinct points in $\mathbb{C} P^{1}$.

If we choose a special hyperbolic lift of a triangulation, we obtain a well defined function (up to a sign) on the 1-simplices of the triangulation. That function recuperates the cross ratio of the 3 -simplices according to the formula above.

\subsection{Bloch identity.}

DeFInItion 4.8. Given a finite hyperbolic triangulation $T=\left(T_{i}, z_{i}\right)$ by ideal tetrahedra, we define its Bloch sum by

$$
\delta(\beta(T))=\sum_{i} z_{i} \wedge\left(1-z_{i}\right) .
$$

We prove

THEOREM 4.9. For a finite ideal triangulation $T$ of a finite volume hyperbolic manifold

$$
\delta(\beta(T))=0 .
$$

Proof. Consider a development of the triangulation with vertices in $\mathbb{C} P^{1}$. There exists a special lift by the Proposition above. We compute the following sum

$$
\sum_{i}\left[p_{0}^{i}, p_{1}^{i}, p_{2}^{i}, p_{3}^{i}\right] \wedge\left[p_{0}^{i}, p_{2}^{i}, p_{3}^{i}, p_{1}^{i}\right] .
$$

For each tetrahedron $\left(p_{0}, p_{1}, p_{2}, p_{3}\right)$, let $v_{i}$ be the lift of $p_{i}$. Then

Therefore

$$
\left[p_{0}, p_{1}, p_{2}, p_{3}\right]=\frac{\left[v_{2}, v_{0}\right]\left[v_{3}, v_{1}\right]}{\left[v_{3}, v_{0}\right]\left[v_{2}, v_{1}\right]} .
$$

$$
\begin{aligned}
& {\left[p_{0}, p_{1}, p_{2}, p_{3}\right] \wedge\left[p_{0}, p_{2}, p_{3}, p_{1}\right]=\frac{\left[v_{2}, v_{0}\right]\left[v_{3}, v_{1}\right]}{\left[v_{3}, v_{0}\right]\left[v_{2}, v_{1}\right]} \wedge \frac{\left[v_{3}, v_{0}\right]\left[v_{1}, v_{2}\right]}{\left[v_{1}, v_{0}\right]\left[v_{3}, v_{2}\right]} } \\
= & {\left[v_{0}, v_{2}\right] \wedge\left[v_{0}, v_{3}\right]+\left[v_{0}, v_{2}\right] \wedge\left[v_{2}, v_{1}\right]-\left[v_{0}, v_{2}\right] \wedge\left[v_{2}, v_{3}\right]-\left[v_{0}, v_{2}\right] \wedge\left[v_{0}, v_{1}\right] } \\
& +\left[v_{1}, v_{3}\right] \wedge\left[v_{0}, v_{3}\right]+\left[v_{1}, v_{3}\right] \wedge\left[v_{2}, v_{1}\right]-\left[v_{1}, v_{3}\right] \wedge\left[v_{2}, v_{3}\right]-\left[v_{1}, v_{3}\right] \wedge\left[v_{0}, v_{1}\right] \\
& -\left[v_{1}, v_{2}\right] \wedge\left[v_{0}, v_{3}\right]-\left[v_{1}, v_{2}\right] \wedge\left[v_{2}, v_{1}\right]+\left[v_{1}, v_{2}\right] \wedge\left[v_{2}, v_{3}\right]+\left[v_{1}, v_{2}\right] \wedge\left[v_{0}, v_{1}\right] \\
& -\left[v_{0}, v_{3}\right] \wedge\left[v_{0}, v_{3}\right]-\left[v_{0}, v_{3}\right] \wedge\left[v_{2}, v_{1}\right]+\left[v_{0}, v_{3}\right] \wedge\left[v_{2}, v_{3}\right]+\left[v_{0}, v_{3}\right] \wedge\left[v_{0}, v_{1}\right] .
\end{aligned}
$$


Observe that $(-a) \wedge b=a \wedge b$ and $[v, w]=-[w, v]$. Therefore we can write

$$
\begin{aligned}
& {\left[p_{0}, p_{1}, p_{2}, p_{3}\right] \wedge\left[p_{0}, p_{2}, p_{3}, p_{1}\right] } \\
= & {\left[v_{0}, v_{1}\right] \wedge\left[v_{0}, v_{2}\right]+\left[v_{0}, v_{2}\right] \wedge\left[v_{0}, v_{3}\right]+\left[v_{0}, v_{3}\right] \wedge\left[v_{0}, v_{1}\right] } \\
& +\left[v_{1}, v_{2}\right] \wedge\left[v_{1}, v_{0}\right]+\left[v_{1}, v_{3}\right] \wedge\left[v_{1}, v_{2}\right]+\left[v_{1}, v_{0}\right] \wedge\left[v_{1}, v_{3}\right] \\
& +\left[v_{2}, v_{0}\right] \wedge\left[v_{2}, v_{1}\right]+\left[v_{2}, v_{1}\right] \wedge\left[v_{2}, v_{3}\right]+\left[v_{2}, v_{3}\right] \wedge\left[v_{2}, v_{0}\right] \\
& +\left[v_{3}, v_{0}\right] \wedge\left[v_{3}, v_{2}\right]+\left[v_{3}, v_{2}\right] \wedge\left[v_{3}, v_{1}\right]+\left[v_{3}, v_{1}\right] \wedge\left[v_{3}, v_{0}\right] .
\end{aligned}
$$

This shows that each face of a fixed tetrahedron appears three times in the sum but the terms corresponding to a common face between two tetrahedra appear with opposite sign. When the faces are identified by a side pairing, the lift of the side pairing, at most, changes the sign of the lifted vertices. But the terms of the sum above are invariant under sign change.

4.3. CR invariant. Let $v_{1}, v_{2} \in \mathbb{C}^{2,1}$ be two vectors in the Hermitian space $\mathbb{C}^{2,1}$ with Hermitian product $\langle\cdot, \cdot\rangle$. We will write $v_{12}=v_{1} \otimes v_{2}$ for the (alternating bilinear) Hermitian cross-product as defined in 2.18. It satisfies, for any $v_{1}, v_{2}, v_{3} \in \mathbb{C}^{2,1}$,

$$
\left\langle v_{1}, v_{23}\right\rangle=\left\langle v_{2}, v_{31}\right\rangle
$$

For four generic points $\left(p_{0}, p_{1}, p_{2}, p_{3}\right)$ in $S^{3}$ and $v_{i} \in \mathbb{C}^{2,1}$ chosen lifts, put

$$
\left[p_{0}, p_{1}, p_{2}, p_{3}\right]=\frac{\left\langle v_{3}, v_{01}\right\rangle\left\langle v_{2}, v_{0}\right\rangle}{\left\langle v_{2}, v_{01}\right\rangle\left\langle v_{3}, v_{0}\right\rangle}
$$

Then by Definition 2.27, 2.24, we have

$$
\beta\left(p_{0}, p_{1}, p_{2}, p_{3}\right)=\left[p_{0}, p_{1}, p_{2}, p_{3}\right]+\left[p_{1}, p_{0}, p_{3}, p_{2}\right]+\left[p_{2}, p_{3}, p_{0}, p_{1}\right]+\left[p_{3}, p_{2}, p_{1}, p_{0}\right] .
$$

The next proposition computes the image of $\beta\left(p_{0}, p_{1}, p_{2}, p_{3}\right)$ under the map

$$
\delta: \mathcal{P}(\mathbb{C}) \rightarrow \mathbb{C}^{*} \wedge \mathbb{C}^{*}
$$

defined on the generators of $\mathcal{P}(\mathbb{C})$ by $\delta([z])=z \wedge(1-z)$. The main objective is to obtain an expression which depends on four oriented surface terms. These terms will cancel out when two configurations have a common face.

LEMMA 4.10. Let $p_{i} \in S^{3}, 0 \leq i \leq 3$, be four pairwise distinct points and $v_{i} \in \mathbb{C}^{2,1}$ chosen lifts (to simplify notations we will denote $v_{i}$ simply $i$ ). We have

$$
\begin{aligned}
-\delta\left(\beta\left(p_{0}, p_{1}, p_{2}, p_{3}\right)\right)= & \langle 3,01\rangle \wedge \frac{\langle 0,1\rangle\langle 1,3\rangle\langle 3,0\rangle}{\langle 0,3\rangle\langle 3,1\rangle\langle 1,0\rangle}+\langle 2,01\rangle \wedge \frac{\langle 0,2\rangle\langle 2,1\rangle\langle 1,0\rangle}{\langle 0,1\rangle\langle 1,2\rangle\langle 2,0\rangle} \\
& +\langle 3,02\rangle \wedge \frac{\langle 0,3\rangle\langle 3,2\rangle\langle 2,0\rangle}{\langle 0,2\rangle\langle 2,3\rangle\langle 3,0\rangle}+\langle 2,31\rangle \wedge \frac{\langle 1,2\rangle\langle 2,3\rangle\langle 3,1\rangle}{\langle 1,3\rangle\langle 3,2\rangle\langle 2,1\rangle} \\
& +\langle 2,0\rangle \wedge\langle 3,0\rangle+\langle 1,0\rangle \wedge\langle 2,0\rangle+\langle 3,0\rangle \wedge\langle 1,0\rangle \\
& +\langle 3,1\rangle \wedge\langle 2,1\rangle+\langle 0,1\rangle \wedge\langle 3,1\rangle+\langle 2,1\rangle \wedge\langle 0,1\rangle \\
& +\langle 0,2\rangle \wedge\langle 1,2\rangle+\langle 3,2\rangle \wedge\langle 0,2\rangle+\langle 1,2\rangle \wedge\langle 3,2\rangle \\
& +\langle 1,3\rangle \wedge\langle 0,3\rangle+\langle 2,3\rangle \wedge\langle 1,3\rangle+\langle 0,3\rangle \wedge\langle 2,3\rangle .
\end{aligned}
$$

Proof. To simplify notation we write

$$
\left[p_{0}, p_{1}, p_{2}, p_{3}\right]=[0,1,2,3]=\frac{\langle 3,01\rangle\langle 2,0\rangle}{\langle 2,01\rangle\langle 3,0\rangle} .
$$


Also, observe that

$$
-\delta([0,1,2,3])=[0,1,2,3] \wedge[0,2,3,1]
$$

Therefore we need to compute

$$
\begin{aligned}
-\delta\left(\beta\left(p_{0}, p_{1}, p_{2}, p_{3}\right)\right)= & {[0,1,2,3] \wedge[0,2,3,1]+[1,0,3,2] \wedge[1,3,2,0] } \\
& +[2,3,0,1] \wedge[2,0,1,3]+[3,2,1,0] \wedge[3,1,0,2] \\
= & \frac{\langle 3,01\rangle\langle 2,0\rangle}{\langle 2,01\rangle\langle 3,0\rangle} \wedge \frac{\langle 1,02\rangle\langle 3,0\rangle}{\langle 3,02\rangle\langle 1,0\rangle}+\frac{\langle 2,10\rangle\langle 3,1\rangle}{\langle 3,10\rangle\langle 2,1\rangle} \wedge \frac{\langle 0,13\rangle\langle 2,1\rangle}{\langle 2,13\rangle\langle 0,1\rangle} \\
& +\frac{\langle 1,23\rangle\langle 0,2\rangle}{\langle 0,23\rangle\langle 1,2\rangle} \wedge \frac{\langle 3,20\rangle\langle 1,2\rangle}{\langle 1,20\rangle\langle 3,2\rangle}+\frac{\langle 0,32\rangle\langle 1,3\rangle}{\langle 1,32\rangle\langle 0,3\rangle} \wedge \frac{\langle 2,31\rangle\langle 0,3\rangle}{\langle 0,31\rangle\langle 2,3\rangle} .
\end{aligned}
$$

We may use the distributive property of the wedge product to obtain a sum of three types of terms. The first type one is of the form $\langle i, j k\rangle \wedge\left\langle i^{\prime}, j^{\prime} k^{\prime}\right\rangle$. The second one is of the form $\langle i, j k\rangle \wedge\langle n, m\rangle$. The third type is $\langle i, j\rangle \wedge\langle n, m\rangle$.

Using the property $\langle 1,23\rangle=\langle 2,31\rangle=-\langle 1,32\rangle$ and the fact that $(-1) \wedge z=0$ for all $z$ we can treat $\langle i, j k\rangle$ as invariant under all permutations.

We obtain by a straightforward computation that all terms of type 1 cancel out. Also, the terms of type 2 can be written in a more concise form as

$$
\begin{aligned}
& \langle 3,01\rangle \wedge \frac{\langle 0,1\rangle\langle 1,3\rangle\langle 3,0\rangle}{\langle 0,3\rangle\langle 3,1\rangle\langle 1,0\rangle}+\langle 2,01\rangle \wedge \frac{\langle 0,2\rangle\langle 2,1\rangle\langle 1,0\rangle}{\langle 0,1\rangle\langle 1,2\rangle\langle 2,0\rangle} \\
& +\langle 3,02\rangle \wedge \frac{\langle 0,3\rangle\langle 3,2\rangle\langle 2,0\rangle}{\langle 0,2\rangle\langle 2,3\rangle\langle 3,0\rangle}+\langle 2,31\rangle \wedge \frac{\langle 1,2\rangle\langle 2,3\rangle\langle 3,1\rangle}{\langle 1,3\rangle\langle 3,2\rangle\langle 2,1\rangle} .
\end{aligned}
$$

Finally, the terms of type 3 are the following

$$
\begin{aligned}
& \langle 2,0\rangle \wedge\langle 3,0\rangle+\langle 1,0\rangle \wedge\langle 2,0\rangle+\langle 3,0\rangle \wedge\langle 1,0\rangle \\
& +\langle 3,1\rangle \wedge\langle 2,1\rangle+\langle 0,1\rangle \wedge\langle 3,1\rangle+\langle 2,1\rangle \wedge\langle 0,1\rangle \\
& +\langle 0,2\rangle \wedge\langle 1,2\rangle+\langle 3,2\rangle \wedge\langle 0,2\rangle+\langle 1,2\rangle \wedge\langle 3,2\rangle \\
& +\langle 1,3\rangle \wedge\langle 0,3\rangle+\langle 2,3\rangle \wedge\langle 1,3\rangle+\langle 0,3\rangle \wedge\langle 2,3\rangle .
\end{aligned}
$$

Consider a CR triangulation and Let $p_{i} \in S^{3}$ be the vertices of the developement map as in the real hyperbolic case. Choose a lift $v_{i} \in \mathbb{C}^{3}$ for each vertex $p_{i}$. The vertices of the faces of the polyhedron are identified by side pairings in $\operatorname{PU}(2,1)$.

DEFinition 4.11. Define the holonomy group of a CR triangulation to be the group generated by the face pairing transformations of the polyhedron.

Definition 4.12. We call a lift of the vertices of a development of a CR triangulation special if there are lifts of the side pairings preserving the lifted vertices up to multiplication by a root of unity.

LEMMA 4.13. If a CR triangulation is such that each vertex of the developed triangulation is the fixed point of purely parabolic elements (or the identity) of the holonomy group then it has a special lift.

Proof. The proof is the same as in the case of real hyperbolic structures. That is, we divide the vertices into classes, each one obtained by translating a fixed vertex 
by elements of the holonomy group. That is, fixing a vertex $p \in S^{3}$ we consider all vertices identified to $p$ by the holonomy group. That is, the other vertices $p_{i}$ are obtained by $p_{i}=g_{i} p$ for $g_{i} \in \Gamma$. In order to verify the compatibility, suppose $g_{i} p=g_{j} p$. Then $g_{j}^{-1} g_{i} p=p$ and by hypothesis $g_{j}^{-1} g_{i}$ is purely parabolic. Without loss of generality, we suppose that $p=\infty$ and let $\hat{g}_{i} \in S U(2,1)$ be a lift of $g_{i}$ and let $v_{i}=\hat{g}_{i} v$. Then $\hat{g}_{i} \hat{p}=\omega g_{j} \hat{p}$ where $\omega$ is a cubic root of unity. Let $g \in \Gamma$ be a side pairing of the polyhedra obtained by the development map. We have $g g_{i} p=g_{j} p$, therefore $\left(g_{j}\right)^{-1} g g_{i} p=p$ and we conclude that $\left(g_{j}\right)^{-1} g g_{i}$ is parabolic and then its lift $\left(\hat{g}_{j}\right)^{-1} \hat{g} \hat{g}_{i}$ is a parabolic element (or the identity element) fixing $v$ up to a cubic root of unity. We conclude that

$$
\hat{g} v_{i}=v_{j}
$$

up to multiplication by a cubic root of unity. We do that for each cycle of vertices and obtain that the lift is special.

THEOREM 4.14. Let $M$ be a 3-dimensional spherical $C R$ manifold with a $C R$ triangulation, say $M=\Delta_{1} \cup \cdots \cup \Delta_{k}$ with each $\Delta_{i}$ a CR tetrahedron. Suppose $M$ is non-compact with purely parabolic boundary. Then its invariant $\beta(M) \in B(\mathbb{C})$, that is,

$$
\sum_{i} \delta\left(\beta\left(\Delta_{i}\right)\right)=0
$$

Proof. The proof follows from the previous proposition. We choose a special lift and each face of a fixed tetrahedron appears four times in the sum but the terms corresponding to a common face between two tetrahedra appear with opposite sign. The only problem might arise with the side pairing maps, but the surface terms obtained will differ by terms (which are null) of the form $\omega \wedge a$ where $\omega$ is a cubic root of unity.

The arguments above are valid if we substitute $\mathbb{C}$ for $k=k_{\mathbf{x}}$ where $k_{\mathbf{x}}$ is the field generated by the cross-ratios of a triangulation. We will make the hypothesis that $k$ is a number field in order to obtain a special lift:

LEMMA 4.15. If a CR triangulation with $k=k_{\mathbf{x}}$ a number field is such that each vertex of the developed triangulation is the fixed point of a parabolic or elliptic element (that is, we exclude loxodromic elements) of the holonomy group then a finite cover of the triangulation has development with a special lift.

Here the root of unity appearing in the definition of a special lift is in $k$.

Proof. We first consider a normalized development of the vertices. By Proposition 4.2 the coordinates of a normalised development of the triangulation are contained in the invariant field. By Lemma 3.4 of [Ge] any element $M$ in the holonomy is such that $M^{3} \in S U(2,1, k)$. Let $\Gamma$ be the group generated by the cubes. It is a finite index subgroup of the holonomy group and we consider the corresponding finite cover of the triangulation. It defines the same field $k$ but now the side pairings have lifts in $S U(2,1, k)$. Now we argue as before: we divide the vertices into classes, each one obtained by translating a fixed vertex by elements of the holonomy group. Fixing a vertex $p \in S^{3}$ we consider all vertices identified to $p$ by the holonomy group. That is, the other vertices $p_{i}$ are obtained by $p_{i}=g_{i} p$ for $g_{i} \in \Gamma$. Suppose $g_{i} p=g_{j} p$. Then $g_{j}^{-1} g_{i} p=p$ and by hypothesis $g_{j}^{-1} g_{i}$ is parabolic or elliptic with coefficients in 
$k$. Without loss of generality, we suppose that $p=\infty$ and let $\hat{g}_{i} \in S U(2,1, k)$ be a lift of $g_{i}$ and $v_{i}=\hat{g}_{i} v$. Then $\hat{g}_{i} \hat{p}=\mu g_{j} \hat{p}$ where $\mu$ is a root of unity in the field $k$. Let $g \in \Gamma$ be a side pairing of the polyhedra obtained by the development map. We have $g g_{i} p=g_{j} p$, therefore $\left(g_{j}\right)^{-1} g g_{i} p=p$ and we conclude that $\left(g_{j}\right)^{-1} g g_{i}$ is parabolic or elliptic and then its lift $\left(\hat{g}_{j}\right)^{-1} \hat{g} \hat{g}_{i}$ fixes $v$ up to a root of unity. We conclude that

$$
\hat{g} v_{i}=v_{j} .
$$

up to multiplication by a root of unity. We do that for each cycle of vertices and obtain that the lift is special.

THEOREM 4.16. Let $k=k_{\mathbf{x}}$ (which we suppose to be a number field) be the invariant field of a $C R$-triangulation of $M$ with parabolic or elliptic boundary holonomy. Then there exists an integer $d \geq 1$ such that $d \beta(M) \in \mathcal{B}(k)$.

Proof. By the proposition above we obtain a special normalized lift of a certain finite cover of $M$. The proof then follows as in theorem 4.14 for the cover so that $d \beta(M) \in \mathcal{B}(k)$ for an integer $d \geq 1$.

TheOREm 4.17. Let $k_{\mathbf{x}}$ be the field of a CR-triangulation $(T, \mathbf{X})$. Suppose $k_{\mathbf{X}} \subset k$ where $k$ is a purely imaginary quadratic extension of a totally real field. If $\beta(T, \mathbf{X}) \in \mathcal{B}(k)$ then it is torsion.

Proof. We proved that $D(\beta(T, \mathbf{X}))=0$ and the result follows from Borel's theorem.

Let $M$ be a CR-triangulation. Assume that its invariant $\beta(M) \in \mathcal{B}(\mathbb{C})$. Then we have $\rho(\beta(M)) \in \mathbb{C} / \pi^{2} \mathbb{Q}$. By Theorem 3.12, we know $\operatorname{Im} \rho(\beta(M))=0$.

Definition 4.18. Let $M$ be as above with $\beta(M) \in \mathcal{B}(\mathbb{C})$. We define its ChernSimons invariant to be the real part of $\rho(\beta(M))$, denoted by $C S(M)$.

REMARK. Theorem 4.17 implies that if the invariant field associated to a $\mathrm{CR}$ structure is an imaginary quadratic extension of a totally real field then $C S(M)=0$.

5. Examples. Besides the 5-term relation (1) at the beginning, it is known ([S1]) that we have two more identities in $\mathcal{P}(\mathbb{C})$ :

$$
\begin{gathered}
{[z]+\left[z^{-1}\right]=0,} \\
{[z]+[1-z]=0 .}
\end{gathered}
$$

In the following, for a comlex number $a$, we will denote its complex conjugation by $\bar{a}$.

5.1. Figure 8 knot complement. The Figure 8 knot complement $K$ can be glued by two ideal CR tetrahedra. Solving the equations in [F2] imposing that $R\left(H_{2}\right)$ be real, we obtain the following one real parameter family of solutions: Let

$$
\alpha=\sqrt{2-4 \beta^{2}+2 \sqrt{5-8 \beta}} .
$$

Observe that $\alpha$ is real for $I=\left\{\beta \mid-\frac{1}{2}-\frac{\sqrt{5}}{2} \leq \beta \leq \frac{5}{8}\right\}$. Then one branch of solutions is given (for $\beta \in I$ ), by:

$$
w_{12}=\beta+\frac{\alpha}{2} i ; \quad w_{21}=\beta-\frac{\alpha}{2} i ; \quad w_{34}=\beta+\frac{\alpha}{2} i ; \quad w_{43}=\beta-\frac{\alpha}{2} i .
$$


and

$$
z_{12}=\frac{\sqrt{5-8 \beta}-2 \beta+1+\alpha i}{\sqrt{5-8 \beta}+3-4 \beta} ; \quad z_{21}=\overline{z_{12}} ; \quad z_{34}=z_{12} ; \quad z_{43}=\overline{z_{12}}
$$

\section{LEMMA 5.1. The invariant}

$$
\left[w_{12}\right]+\left[w_{21}\right]+\left[w_{34}\right]+\left[w_{43}\right]+\left[z_{12}\right]+\left[z_{21}\right]+\left[z_{34}\right]+\left[z_{43}\right]=0 \text { in } \mathcal{P}(\mathbb{C}) .
$$

Proof. By the definition, we see that $\mathrm{LHS}=2\left(\left[w_{12}\right]+\left[\overline{w_{12}}\right]+\left[z_{12}\right]+\left[\overline{z_{12}}\right]\right)$. We will check that

$$
\frac{1}{1-\frac{1}{w_{12}}}=\frac{w_{12}}{w_{12}-1}=\overline{z_{12}}, \text { and } \frac{1}{1-\frac{1}{w_{12}}}=\frac{\overline{w_{12}}}{\overline{w_{12}}-1}=z_{12} .
$$

Then it will follow that $\left[w_{12}\right]+\left[\overline{z_{12}}\right]=0$, and $\left[\overline{w_{12}}\right]+\left[z_{12}\right]=0$. The lemma will be proved. We only need to check the first one since the second is the complex conjugation of the first one.

$$
\frac{w_{12}}{w_{12}-1}=\frac{\beta+\frac{\alpha}{2} i}{(\beta-1)+\frac{\alpha}{2} i}=\frac{\left(\beta+\frac{\alpha}{2} i\right)\left((\beta-1)-\frac{\alpha}{2} i\right)}{(\beta-1)^{2}+\frac{\alpha^{2}}{4}}=\frac{\left[\beta(\beta-1)+\frac{\alpha^{2}}{4}-\frac{\alpha}{2} i\right]}{(\beta-1)^{2}+\frac{\alpha^{2}}{4}} .
$$

Multiplying by 2 on the numerator and denominator of the last fraction, we get

$$
\frac{w_{12}}{w_{12}-1}=\frac{2 \beta(\beta-1)+\frac{\alpha^{2}}{2}-\alpha i}{2(\beta-1)^{2}+\frac{\alpha^{2}}{2}} .
$$

By the equation of $\alpha$ and $\beta$ at the beginning, we have

$$
2 \beta^{2}+\frac{\alpha^{2}}{2}=1+\sqrt{5-8 \beta}
$$

Plug this in and simplify, we have

$$
\frac{w_{12}}{w_{12}-1}=\frac{(\sqrt{5-8 \beta}-2 \beta+1)-\alpha i}{\sqrt{5-8 \beta}+3-4 \beta}=\overline{z_{12}} \text {. }
$$

For $\beta=1 / 2$ we obtain $\alpha=\sqrt{3}$ and therefore the invariant

$$
\beta(K)=4([\omega]+[\bar{\omega}]),
$$

where $\omega=\exp \left(\frac{2 \pi i}{3}\right)$ is a primitive cube root of unity. Since $\bar{\omega}=\omega^{-1}$, by (5), we see in $\mathcal{P}(\mathbb{C})$

$$
[\omega]+[\bar{\omega}]=0
$$

So $\beta(K)=0$.

In [F2, Page 94], there are two other representations from the fundamental group of the figure eight complement to $P U(2,1)$. They have cyclic holonomy on the boundary. The first invariants are

$$
w_{12}=\frac{3}{8}+\frac{\sqrt{7}}{8} i ; \quad w_{21}=\frac{5}{4}+\frac{\sqrt{7}}{4} i ; \quad w_{34}=\frac{3}{8}-\frac{\sqrt{7}}{8} i ; \quad w_{43}=\frac{5}{4}-\frac{\sqrt{7}}{4} i .
$$


The second invariants are

$$
t_{12}=\frac{3}{2}+\frac{\sqrt{7}}{2} i ; \quad t_{21}=-\frac{1}{4}-\frac{\sqrt{7}}{4} i ; \quad t_{34}=\frac{3}{2}-\frac{\sqrt{7}}{2} i ; \quad t_{43}=-\frac{1}{4}+\frac{\sqrt{7}}{4} i .
$$

Let $F=\mathbb{Q}(\sqrt{-7})$. Then we have the invariants

$\beta_{1}(K)=2\left(\left[w_{12}\right]+\left[w_{21}\right]+\left[w_{34}\right]+\left[w_{43}\right]\right), \quad \beta_{2}(K)=2\left(\left[t_{12}\right]+\left[t_{21}\right]+\left[t_{34}\right]+\left[t_{43}\right]\right) \in \mathcal{P}(F)$.

Proposition 5.2. (1). $\beta_{1}(K)=-2 c_{F}$ in $\mathcal{P}(F)$.

(2). $\beta_{2}(K)=0$ in $\mathcal{P}(F)$.

(3). $\beta_{1}(K) \in \mathcal{B}(F)$ is a non-trivial torsion of order 3 .

Proof. (1). Let's put

$$
a=w_{34}=\frac{3}{8}-\frac{\sqrt{7}}{8} i, \quad b=w_{43}=\frac{5}{4}-\frac{\sqrt{7}}{4} i,
$$

then

$$
\frac{1}{2} b=\frac{5}{8}-\frac{\sqrt{7}}{8} i=1-\bar{a}, \quad \frac{1}{2} \bar{b}=\frac{5}{8}+\frac{\sqrt{7}}{8} i=1-a,
$$

and

$$
[\bar{a}]=c_{F}-[1-\bar{a}]=c_{F}-\left[\frac{1}{2} b\right], \quad[a]=c_{F}-[1-a]=c_{F}-\left[\frac{1}{2} \bar{b}\right]
$$

Therefore,

$$
\beta_{1}(K)=2\left([b]+[\bar{b}]-\left[\frac{1}{2} b\right]-\left[\frac{1}{2} \bar{b}\right]+2 c_{F}\right) .
$$

By the five-term equation (1), take $x=\frac{1}{2}, y=\frac{1}{2} b$, then we obtain

$$
\left[\frac{1}{2}\right]-\left[\frac{1}{2} b\right]+[b]-\left[\frac{1-2}{1-\left(\frac{1}{2} b\right)^{-1}}\right]+\left[\frac{1-\frac{1}{2}}{1-\frac{1}{2} b}\right]=0 .
$$

Direct computations show that

$$
s=\frac{1-\frac{1}{2}}{1-\frac{1}{2} b}=\frac{3}{4}-\frac{\sqrt{7}}{4} i, \text { and } \frac{1-2}{1-\left(\frac{1}{2} b\right)^{-1}}=\frac{1}{\frac{1}{4}+\frac{\sqrt{7}}{4} i}=\frac{1}{1-s} .
$$

Hence,

$$
[b]-\left[\frac{1}{2} b\right]=\left[\frac{1}{1-s}\right]-[s]-\left[\frac{1}{2}\right] .
$$

Similarly by taking $x=\frac{1}{2}, y=\frac{1}{2} \bar{b}$, we have

$$
[\bar{b}]-\left[\frac{1}{2} \bar{b}\right]=\left[\frac{1}{1-\bar{s}}\right]-[\bar{s}]-\left[\frac{1}{2}\right] .
$$

Hence,

$$
\beta_{1}(K)=2\left(\left[\frac{1}{1-s}\right]-[s]+\left[\frac{1}{1-\bar{s}}\right]-[\bar{s}]-c_{F}\right)+4 c_{F}=2\left(\left[\frac{1}{1-s}\right]-[s]+\left[\frac{1}{1-\bar{s}}\right]-[\bar{s}]\right)+2 c_{F} .
$$


By Lemma 1.2 of $[\mathrm{S} 1]$, in $\mathcal{P}(F)$ we have

$$
2\left(\left[\frac{1}{1-s}\right]\right)=-2[1-s], \text { and } 2\left(\left[\frac{1}{1-\bar{s}}\right]\right)=-2[1-\bar{s}]
$$

so

$$
\beta_{1}(K)=-2 c_{F}-2 c_{F}+2 c_{F}=-2 c_{F}
$$

(2). Notice that

$$
t_{43}=1-w_{43}, \quad t_{21}=1-w_{21}, t_{12}=w_{34}^{-1} ; t_{34}=w_{12}^{-1} .
$$

Hence we have

$$
\left[t_{43}\right]=c_{F}-\left[w_{43}\right], \quad\left[t_{21}\right]=c_{F}-\left[w_{21}\right], \quad 2\left[t_{12}\right]=-2\left[w_{34}\right], \quad 2\left[t_{34}\right]=-2\left[w_{12}\right],
$$

and

$$
\beta_{2}(K)=2\left(\left[t_{12}\right]+\left[t_{21}\right]+\left[t_{34}\right]+\left[t_{43}\right]\right)=4 c_{F}-\beta_{1}(K)=6 c_{F}=0 .
$$

The last equality comes from [S1, Lemma 1.5(a)].

(3). Since $c_{F} \in \mathcal{B}(F)$, by $(1), \beta_{1}(K) \in \mathcal{B}(F)$. It is easy to see that for $F=\mathbb{Q}(\sqrt{-7}), \mu(F)=\{ \pm 1\}=\mathbb{Z} / 2$. Hence $\operatorname{Tor}(\mu(F), \mu(F))=\mathbb{Z} / 2$. The result follows by Lemma 2.7 .

Corollary 5.3. Both $\beta_{1}(K)$ and $\beta_{2}(K)$ are zero in $\mathcal{B}(\mathbb{C})$.

Proof. $\beta_{2}(K)=0$ in $\mathcal{B}(\mathbb{C})$ since it is already zero in $\mathcal{P}(F)$. For $\beta_{1}(K)$, since it is a torsion in $\mathcal{B}(F)$, it will be a torsion in $\mathcal{B}(\mathbb{C})$. We know that $\mathcal{B}(\mathbb{C})$ is torsion-free, hence $\beta_{1}(K)=0$ in $\mathcal{B}(\mathbb{C})$.

5.2. Whitehead link complement. The Whitehead link complement $W$ can be glued by four ideal CR tetrahedra. See [Sc, Ge]. Their CR invariants are:

$$
\begin{gathered}
A_{01}=-\frac{1}{8}+\frac{\sqrt{15}}{8} i ; \quad A_{10}=-2 ; \quad A_{23}=-\frac{3}{4}+\frac{\sqrt{15}}{4} i ; \quad A_{32}=\frac{1}{2}-\frac{\sqrt{15}}{6} i \\
B_{01}=-2 ; \quad B_{10}=\bar{A}_{01} ; \quad B_{23}=\bar{A}_{32} ; \quad B_{32}=\bar{A}_{23} . \\
C_{01}=\bar{A}_{01} ; \quad C_{10}=-2 ; \quad C_{23}=\bar{A}_{23} ; \quad C_{32}=\bar{A}_{32} . \\
D_{01}=-2 ; \quad D_{10}=A_{01} ; \quad D_{23}=A_{32} ; \quad D_{32}=A_{23} .
\end{gathered}
$$

Let $F=\mathbb{Q}(\sqrt{-15})$. Then we have

$$
\beta(W)=4[-2]+2\left(\left[A_{01}\right]+\left[\bar{A}_{01}\right]+\left[A_{23}\right]+\left[\bar{A}_{23}\right]+\left[A_{32}\right]+\left[\bar{A}_{32}\right]\right) \in \mathcal{P}(F) .
$$

In $\mathcal{P}(F)$, by $[\mathrm{S} 1$, Lemma 1.2$]$, for any $z \neq 0,1$ we have :

$$
2\left([z]+\left[z^{-1}\right]\right)=0 .
$$


Lemma 5.4. Let $z \in F-\{0,1,-1\}$. Then we have

$$
2\left[z^{2}\right]=4[z]+4[-z] .
$$

Proof. By $\left[\right.$ S1, Lemma 4.5], we have $\left[z^{2}\right]=2([z]+[-z]+[-1])$. By (7), we have $4[-1]=0$. Hence

$$
2\left[z^{2}\right]=4[z]+4[-z]+4[-1]=2\left[z^{2}\right]=4[z]+4[-z] .
$$

Proposition 5.5. (1). $\beta(W)=4\left[\frac{1}{2}\right]$ in $\mathcal{P}(F)$.

(2). $\beta(W) \wedge(1-\beta(W))=0$, hence $\beta(W) \in \mathcal{B}(F)$.

(3). $\beta(W)$ has order 3 in $\mathcal{B}(F)$.

Proof. (1). Since $1-A_{32}=\frac{1}{2}+\frac{\sqrt{15}}{6} i=\bar{A}_{32}$, by Definition 2.5, we get

$$
\left[A_{32}\right]+\left[\bar{A}_{32}\right]=\left[A_{32}\right]+\left[1-A_{32}\right]=c_{F} .
$$

In the 5 -term equation (1), take $x=A_{01}, y=A_{01} \bar{A}_{01}=\left|A_{01}\right|^{2}$, we obtain in $\mathcal{P}(F)$ :

$$
\left[A_{01}\right]-\left[\left|A_{01}\right|^{2}\right]+\left[\bar{A}_{01}\right]-\left[\frac{1-A_{01}^{-1}}{1-\left|A_{01}\right|^{-2}}\right]+\left[\frac{1-A_{01}}{1-\left|A_{01}\right|^{2}}\right]=0 .
$$

Therefore,

$$
\left[A_{01}\right]+\left[\bar{A}_{01}\right]=\left[\left|A_{01}\right|^{2}\right]+\left[\frac{1-A_{01}^{-1}}{1-\left|A_{01}\right|^{-2}}\right]-\left[\frac{1-A_{01}}{1-\left|A_{01}\right|^{2}}\right] .
$$

Direct computations show that

$$
\begin{aligned}
& \frac{1-A_{01}^{-1}}{1-\left|A_{01}\right|^{-2}}=A_{23}^{-1} . \\
& \frac{1-A_{01}}{1-\left|A_{01}\right|^{2}}=1-\frac{1}{\bar{A}_{23}} .
\end{aligned}
$$

Therefore,

$$
\left[A_{01}\right]+\left[\bar{A}_{01}\right]=\left[\frac{1}{4}\right]+\left[A_{23}^{-1}\right]-\left[1-\frac{1}{\bar{A}_{23}}\right] .
$$

We obtain that

$$
\begin{aligned}
\beta(W) & =4[-2]+2\left(\left[A_{01}\right]+\left[\bar{A}_{01}\right]+\left[A_{23}\right]+\left[\bar{A}_{23}\right]+\left[A_{32}\right]+\left[\bar{A}_{32}\right]\right) \\
& =4[-2]+2\left(\left[\frac{1}{4}\right]+\left[A_{23}^{-1}\right]-\left[1-\frac{1}{\bar{A}_{23}}\right]+\left[A_{23}\right]+\left[\bar{A}_{23}\right]+c_{F}\right) \\
& =4[-2]+2\left(\left[\frac{1}{4}\right]+\left[A_{23}^{-1}\right]-\left[1-\frac{1}{\bar{A}_{23}}\right]-\left[\frac{1}{\bar{A}_{23}}\right]+\left[\frac{1}{\bar{A}_{23}}\right]+\left[A_{23}\right]+\left[\bar{A}_{23}\right]+c_{F}\right) \\
& =4[-2]+2\left(\left[\frac{1}{4}\right]+\left[A_{23}^{-1}\right]-c_{F}+\left[\frac{1}{\bar{A}_{23}}\right]+\left[A_{23}\right]+\left[\bar{A}_{23}\right]+c_{F}\right) \\
& =4[-2]+2\left(\left[\frac{1}{4}\right]+\left[A_{23}\right]+\left[A_{23}^{-1}\right]+\left[\bar{A}_{23}\right]+\left[\left(\bar{A}_{23}\right)^{-1}\right]\right) \\
& =4[-2]+2\left[\frac{1}{4}\right] \quad(\mathrm{By}(7)) .
\end{aligned}
$$


Next by Lemma 5.4 and $(7)$, we have in $\mathcal{P}(F)$

$$
2\left[z^{2}\right]=4[z]+4[-z] \text { and } 2[z]=-2\left[z^{-1}\right] .
$$

Therefore,

$$
2\left[\frac{1}{4}\right]=4\left[\frac{1}{2}\right]+4\left[-\frac{1}{2}\right]=4\left[\frac{1}{2}\right]-4[-2] .
$$

Now in $\mathcal{P}(F)$ we have

$$
\beta(W)=4[-2]+4\left[\frac{1}{2}\right]-4[-2]=4\left[\frac{1}{2}\right] .
$$

(2). Since

$$
\delta\left(\rho(W)=4\left(\frac{1}{2} \wedge\left(1-\frac{1}{2}\right)\right)=4\left(\frac{1}{2} \wedge \frac{1}{2}\right)=0,\right.
$$

$\beta(W) \in \mathcal{B}(F)$.

(3). Notice $\beta(W)=4\left[\frac{1}{2}\right]=2\left(\left[\frac{1}{2}\right]+\left[1-\frac{1}{2}\right]\right)=2 c_{F}$. It is easy to see that for $F=\mathbb{Q}(\sqrt{-15}), \mu(F)=\{ \pm 1\}=\mathbb{Z} / 2$. Hence $\operatorname{Tor}(\mu(F), \mu(F))=\mathbb{Z} / 2$ and it has no element of order 3. By Lemma 2.7, $\beta(W)=2 c_{F}$ is a nonzero 3-torsion in $\mathcal{B}(F)$.

Corollary 5.6. $\beta(W)=0$ in $\mathcal{B}(\mathbb{C})$.

Proof. It follows from the fact that $\mathcal{B}(\mathbb{C})$ is torsion-free. $\square$

5.3. The $5_{2}$ knot. We thank P.-V. Koseleff for help with the following example. The complement of the $5_{2}$ knot is triangulated using three simplices with parameters $u_{i j}, v_{i j}$ and $w_{i j}$. The edge conditions are:
1. $u_{42} v_{32} u_{34} v_{43} w_{32}=1$
2. $u_{43} v_{23} u_{24} w_{23} v_{34}=1$
3. $u_{31} v_{31} w_{24} v_{21} u_{41} w_{31}=1$
4. $w_{13} u_{14} v_{12} w_{42} v_{13} u_{13}=1$
5. $w_{21} u_{21} w_{41} v_{14} w_{43} u_{23} v_{24}=1$
6. $u_{12} w_{12} v_{42} u_{32} w_{34} v_{41} w_{14}=1$

The face conditions are:
1. $v_{41} v_{31} v_{21} u_{31} u_{41} u_{31}=1$
2. $v_{34} v_{24} v_{14} u_{32} u_{42} u_{12}=1$
3. $u_{43} u_{23} u_{13} w_{34} w_{24} u_{14}=1$
4. $u_{34} u_{14} u_{24} w_{32} w_{42} w_{12}=1$
5. $v_{42} v_{32} v_{12} w_{31} w_{21} w_{41}=1$
6. $v_{43} v_{23} v_{13} w_{13} w_{43} w_{23}=1$

The holonomy equations for a unipotent solution are:

1. $v_{42} \frac{1}{u_{34}} v_{31} \frac{1}{w_{23}} u_{21} \frac{1}{w_{43}} v_{13} \frac{1}{u_{14}} w_{12}=1$

2. $v_{14} \frac{1}{w_{42}}=1$

We have to add 9 conjugacy equations to obtain a CR solution to the equations. Observe that the solutions to the equations above (without the conjugacy 
equations) contain the hyperbolic solution. In particular each solution $X$ to the equation $x^{3}-x+1=0$ give solutions to the equations above making

$$
\begin{aligned}
& u_{12}=X^{2}+X, u_{13}=X^{2}, u_{14}=X, u_{21}=X^{2}+X, u_{23}=X, u_{24}=X^{2}, \\
& u_{31}=X^{2}, u_{32}=X, u_{34}=X^{2}+X, u_{41}=X, u_{42}=X^{2}, u_{43}=X^{2}+X, \\
& v_{12}=X^{2}, v_{13}=X, v_{14}=X^{2}+X, v_{21}=X^{2}, v_{23}=X^{2}+X, v_{24}=X, \\
& v_{31}=X, v_{32}=X^{2}+X, v_{34}=X^{2}, v_{41}=X^{2}+X, v_{42}=X, v_{43}=X^{2}, \\
& w_{12}=X, w_{13}=X^{2}+X, w_{14}=X^{2}, w_{21}=X, w_{23}=X^{2}, w_{24}=X^{2}+X \\
& w_{31}=X^{2}+X, w_{32}=X^{2}, w_{34}=X, w_{41}=X^{2}, w_{42}=X^{2}+X, w_{43}=X
\end{aligned}
$$

Observe that there are two complex conjugate solutions, both being hyperbolic solutions with opposite orientation and the third solution also is CR. Let $F=\mathbb{Q}(X)$ where $X$ is the real solution. From the definition, add together the invariants above, we have $\beta=12\left(\left[X^{2}+X\right]+[X]+\left[X^{2}\right]\right) \in \mathcal{P}(F)$, and $X$ satisfies the equation $x^{3}-x+1=0$.

Lemma 5.7. $\beta$ is in the Bloch group $\mathcal{B}(F)$.

Proof. We need to show that in $\bigwedge^{2} F^{*}$

$$
\delta(\beta)=12\left\{X \wedge(1-X)+X^{2} \wedge\left(1-X^{2}\right)+\left(X^{2}+X\right) \wedge\left(1-X^{2}-X\right)\right\}=0 .
$$

Since $1-X=-X^{3}$, we see that $X \wedge(1-X)=X \wedge(-1)+3 X \wedge X$. Since each term on the right-hand is 2-torsion, $12 X \wedge(1-X)=0$. Next, since $X\left(1-X^{2}\right)=1$, $1-X^{2}=X^{-1}$. Hence, $X^{2} \wedge\left(1-X^{2}\right)=X^{2} \wedge X^{-1}=-2 X \wedge X=0$ and $12 X^{2} \wedge\left(1-X^{2}\right)=0$. Since $1-X^{2}=X^{-1}$, we obtain

$$
\begin{aligned}
\left(X^{2}+X\right) \wedge\left(1-X^{2}-X\right) & =\left(X^{2}+X\right) \wedge\left(X^{-1}-X\right) \\
& =\left(X^{2}+X\right) \wedge \frac{1-X^{2}}{X} \\
& =X(X+1) \wedge X^{-2} \\
& =X \wedge X^{-2}+(X+1) \wedge X^{-2} .
\end{aligned}
$$

Hence $12\left(X^{2}+X\right) \wedge\left(1-X^{2}-X\right)=-24(X+1) \wedge X$ and $\delta(\beta)=24 X \wedge(1+X)$. Now observe that

$$
1=X-X^{3}=X(1-X)(1+X),
$$

therefore $0=X \wedge X(1-X)(1+X)=X \wedge X+X \wedge(1-X)+X \wedge(1+X)$. We conclude that $2 X \wedge(1+X)=0$ and $\beta \in \mathcal{B}(F)$.

Proposition 5.8. $\beta$ has infinite order in $\mathcal{B}(F)$.

Proof. $F=\mathbb{Q}(X)$ has one real embedding and two complex (conjugate) embeddings. Let's fix one complex embedding $\sigma: F \rightarrow \mathbb{C}$. Consider the Borel regulator map: $r: \mathcal{B}(F) \rightarrow \mathbb{R}$. By Borel's Theorem, it suffices to show that $D(\sigma(\beta)) \neq 0$, where $D$ is the dilogarithm function. Since the two complex conjugate solutions to the equation $x^{3}-x+1=0$ correspond to the complete hyperbolic structure on the complement of the knot $5_{2}, D(\sigma(\beta))$ equals a non-zero multiple of the hyperbolic volume, which is clearly non-zero. 
Acknowledgements. E. Falbel thanks Francis Brown, Herbert Gangl, Julien Grivaux and Julien Marché for several helpful conversions. Part of the work was done when Q. Wang visited University Paris VI in January 2010. He thanks University Paris VI for hospitality and support. Q. Wang is grateful to Stephen Lichtenbaum for stimulating discussions.

\section{REFERENCES}

[A] J. W. Alexander, The combinatorial theory of complexes, Ann. of Math. (2), 31:2 (1930), pp. 292-320.

[B] S. BLOCH, Applications of the dilogarithm function in algebraic $K$-theory and algebraic geometry, in: Proc. of the International Symp. on Alg. Geom., Tokyo, 1978.

[B1] S. BLOCH, Higher regulators, algebraic $K$-theory, and zeta functions of elliptic curves, CRM Monograph Series, 11. American Mathematical Society, Providence, RI, 2000.

[Bo] A. Borel, Cohomologie de $\mathrm{SL}_{n}$ et valeurs de fonctions zeta aux points entiers, Ann. Scuola Norm. Sup. Pisa Cl. Sci. (4), 4:4 (1977), pp. 613-636.

[BS] D. Burns And S. Shnider, Spherical Hypersurfaces in Complex Manifolds, Invent. Math., 33 (1976), pp. 223-246.

[C] E. CARTAN, Sur le groupe de la géométrie hypersphérique, Comm. Math. Helv., 4 (1932), pp. $158-171$

[Ca] B. G. CASLER, An imbedding theorem for connected 3-manifolds with boundary, Proc. Amer. Math. Soc., 16 (1965), pp. 559-566.

[CS] S. S. Chern And J. Simons, Characteristic forms and geometric invariants, Ann. of Math. (2), 99 (1974), pp. 48-69.

[D] J. Dupont, The dilogarithm as a characteristic class for flat bundles, Journal of pure and applied algebra, 44 (1987), pp. 137-164.

[DS] J. Dupont And C. H. SAh, Scissors congruences. II, J. Pure Appl. Algebra, 25:2 (1982), pp. 159-195.

[DZ] J. Dupont And C. K. Zickert, A dilogarithm formula for the Cheeger-Chern-Simons class, Geometry and Topology, 10 (2006), pp. 1347-1372.

[F1] E. FALBEL, Geometric structures associated to triangulations as fixed point sets of involutions, Topology and its Applications, 154:6 (2007), pp. 1041-1052.

[F2] E. FALBEL, A spherical CR structure on the complement of the figure eight knot with discrete holonomy, Journal of Differential Geometry, 79 (2008), pp. 69-110.

[F3] E. Falbel, A volume function for Spherical CR Tetrahedra, Quarterly Journal of Mathematics, 2 (2011), pp. 397-415.

[FP] E. Falbel and I. D. Platis, The PU $(2,1)$ configuration space of four points in $S^{3}$ and the cross-ratio variety, Math. Ann., 340:4 (2008), pp. 935-962.

[Ge1] J. Genzmer, Trace fields of subgroups of $S U(n, 1)$, to appear in Acta Math. Viet.

[Ge] J. Genzmer, Sur les triangulations des structures CR sphériques, Thesis, Paris VI (2010).

[G] W. M. Goldman, Complex Hyperbolic Geometry, Oxford Mathematical Monographs. Oxford University Press (1999).

[J] H. JасовоwITZ, An Introduction to CR Structures, Mathematical Surveys and Monographs, 32, American Math. Soc. (1990).

[Ma] S. V. Matveev, Transformations of special spines, and the Zeeman conjecture, Izv. Akad. Nauk SSSR Ser. Mat., 51:5 (1987), pp. 1104-1116, 1119; translation in Math. USSR-Izv., 31:2 (1988), pp. 423-434.

[Ma1] S. V. Matveev, Algorithmic topology and classification of 3-manifolds, Algorithms and Computation in Mathematics, 9. Springer, Berlin, 2007.

[Mc] B. McReYnolds, Arithmetic lattices in PU(2,1), preprint.

[MS] A. S. MerkurJev and A. A. Suslin, The group $K_{3}$ for a field, Math. USSR. Izvestiya, 36:3 (1991), pp. 541-565.

[M] J. MiLnor, Hyperbolic geometry: the first 150 years, Bull. Am. Math. Soc., 6 (1982), pp. 924.

[NR] W. Neumann and A. Reid, Arithmetic of hyperbolic manifolds, Topology '90 (Columbus, OH, 1990), pp. 273-310, Ohio State Univ. Math. Res. Inst. Publ., 1, de Gruyter, Berlin, 1992.

[NZ] W. Neumann And D. Zagier, Volumes of hyperbolic three-manifolds, Topology, 24:3 (1985), pp. 307-332.

[NY1] W. Neumann And J. YAng, Rationality problems for K-theory and Chern-Simons invariants 
of hyperbolic 3-manifolds, Enseign. Math. (2), 41:3-4 (1995), pp. 281-296.

[NY] W. Neumann And J. YAng, Bloch invariants of hyperbolic 3-manifolds, Duke Math. Journal, 96 (1999), pp. 25-59.

[P] U. PACHNER, PL homeomorphic manifolds are equivalent by elementary shellings, Europ. J. Combinatorics, 12 (1991), pp. 129-145.

[PP $]$ J. R. Parker And I. D. Platis, Complex hyperbolic Fenchel-Nielsen coordinates, Topology, 47:2 (2008), pp. 101-135.

[PP1] J. R. PARKER AND I. D. Platis, Global, geometrical coordinates on Falbel's cross-ratio variety, Canadian Mathematical Bulletin, 52 (2009), pp. 285-294.

[R] A. ReID, A note on trace-fields of Kleinian groups, Bull. London Math. Soc., 22:4 (1990), pp. 349-352.

[Sah] C. H. SAH, Homology of classical Lie groups made discrete. III, J. Pure Appl. Algebra, 56:3 (1989), pp. 269-312.

[Sc] R. E. Schwartz, Spherical CR Geometry and Dehn Surgery, Annals of Math. Studies, 165 (2007).

[S1] A. A. SusLin, $K_{3}$ of a field and the Bloch group, Proc. of the Steklov Institute of Math., 4 (1991), pp. 217-238.

[T] W. Thurston, The geometry and topology of 3-manifolds, Lecture notes 1979.

[W1] P. WilL, Traces, cross-ratios and 2-generator subgroups of PU(2,1), Canad. J. Math., 61:6 (2009), pp. 1407-1436.

[W2] P. Will, Bending Fuchsian representations of fundamental groups of cusped surfaces in PU $(2,1)$, J. Differential Geom., 90:3 (2012), pp. 473-520.

[Z] D. ZAGIER, The dilogarithm function, Frontiers in number theory, physics and geometry. II, 3-65, Springer, Berlin, 2007. 